INL/EXT-21-64633 Rev. 0

September 2021

\title{
Atomistic and mesoscale simulations to determine effective diffusion coefficient of fission products in SiC
}

Chao Jiang Jia-Hong Ke Pierre-Clément A. Simon Wen Jiang Larry K. Aagesen

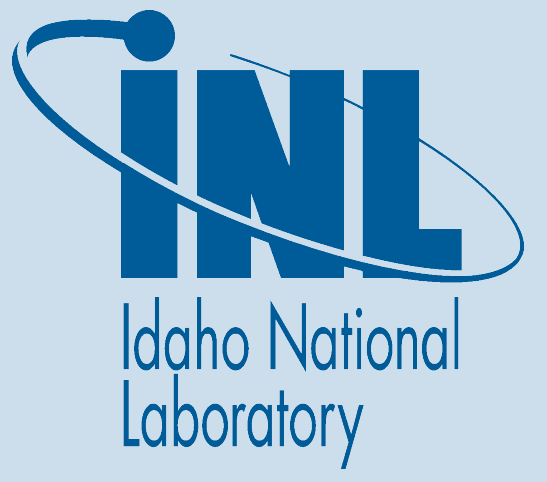




\section{NOTICE}

This information was prepared as an account of work sponsored by an agency of the U.S. Government. Neither the U.S. Government nor any agency thereof, nor any of their employees, makes any warranty, express or implied, or assumes any legal liability or responsibility for any third party's use, or the results of such use, of any information, apparatus, product, or process disclosed herein, or represents that its use by such third party would not infringe privately owned rights. The views expressed herein are not necessarily those of the U.S. Nuclear Regulatory Commission. 
INL/EXT-21-64633 Rev. 0

Atomistic and mesoscale simulations to determine effective diffusion coefficient of fission products in SiC

\author{
Chao Jiang \\ Jia-Hong Ke \\ Pierre-Clément A. Simon \\ Wen Jiang \\ Larry K. Aagesen \\ Idaho National Laboratory \\ Computational Mechanics and Materials Department \\ Idaho Falls, Idaho 83415
}

September 2021

Prepared for the

U.S. Department of Energy

Office of Nuclear Energy

Under U.S. Department of Energy-Idaho Operations Office

Contract DE-AC07-99ID13727 


\begin{abstract}
The silicon carbide ( $\mathrm{SiC}$ ) layer in tristructural isotropic (TRISO) particles serves as the barrier to prevent escape of fission products produced in the fuel kernel. Knowing the diffusion coefficient of fission products through $\mathrm{SiC}$ is critical to determining whether fission gas can escape from the particle. It has been observed in experiments that $\mathrm{Ag}$ accumulated in grain boundaries and triple junctions in $\mathrm{SiC}$. It is hypothesized that grain boundary diffusion is the primary pathway by which fission products penetrate the $\mathrm{SiC}$ layer. In this report, the effective diffusion coefficient of the fission product Ag through the grain boundary network is calculated using a combination of atomistic and phase-field methods. The grain boundary diffusion coefficient is calculated using molecular dynamics simulations. The bulk diffusion coefficient is determined using a combination of density functional theory and nudged elastic band methods. An effective diffusion coefficient is calculated, accounting for the grain structure using a phase-field method. The effective diffusion coefficient will be incorporated into Bison and fission product release calculations are compared to available experimental data.
\end{abstract}




\section{CONTENTS}

FIGURES

$\begin{array}{lll}\text { TABLES } & \text { ix }\end{array}$

1 INTRODUCTION 1

2 ATOMISTIC MODELING OF FISSION PRODUCT DIFFUSION IN BULK $\mathrm{SiC} \quad 2$

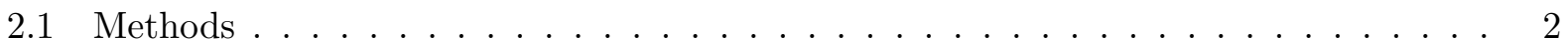

2.1 .1 Multifrequency diffusion model . . . . . . . . . . . . . . . . . 2

2.1.2 First-Principles Methodologies _. . . . . . . . . . . . . . . 5

2.1.3 Kinetic Monte Carlo Methods . . . . . . . . . . . . . . . . . . . . 5

2.2 Density Functional Theory Calculation Results . . . . . . . . . . . . . . . . 6

2.3 Kinetic Monte Carlo Simulation Results . . . . . . . . . . . . . . . . 8

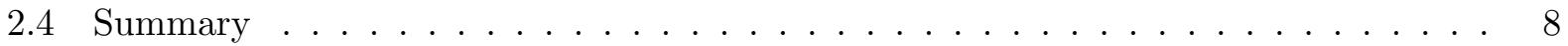

3 MOLECULAR DYNAMICS SIMULATIONS OF Ag DIFFUSION IN SiC 11

3.1 Ag Diffusion Along Grain Boundaries . . . . . . . . . . . . . . . . 11

3.2 Interstitial Ag Diffusion in Bulk Crystalline $\mathrm{SiC} \ldots \ldots \ldots$. . . . . . . . . . 12

3.3 Modeling Ag Diffusion in Bulk SiC Using Temperature-accelerated Dynamics . . . . 12

4 EFFECTIVE DIFFUSIVITY OF Ag VIA POLYCRYSTALLINE SiC 19

4.1 Scaling Up of the Microstructure for Effective Diffusivity Calculations . . . . . . . . 19

4.1.1 Analytical Expression of the Effective Diffusion Coefficient for Simple Mi-

crostructures . . . . . . . . . . . . . . . . . . 19

4.1.2 Change in the Effective Diffusion Coefficient when the GB Width is Increased 21

4.1.3 Verification on Simple Bicrystals with Sharp Interfaces . . . . . . . . . . . . . 22

4.1.4 Verification on Polycrystals with Continuous Interfaces . . . . . . . . . . . . . 23

4.1.5 Effective Diffusivity Calculations . . . . . . . . . . . . . . . . 23

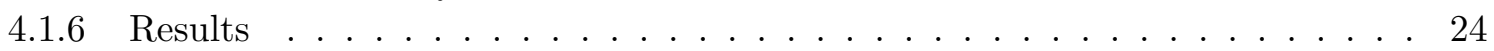

4.2 Mesoscale effective $\mathrm{Ag}$ diffusivity in polycrystalline $\mathrm{SiC} \ldots \ldots \ldots \ldots$

4.2.1 Method: Generate Polycrystalline $\mathrm{SiC} \ldots \ldots \ldots \ldots$

4.2.2 Method: Effective Diffusivity Calculations . . . . . . . . . . . . . . 25

4.2 .3 Results . . . . . . . . . . . . . . . . . . 26

4.3 Implementation of the Effective Ag Diffusivity in Bison . . . . . . . . . . 28

4.3 .1 Methods . . . . . . . . . . . . . . . . . . . 28

4.3 .2 Results . . . . . . . . . . . . . . . . . 28

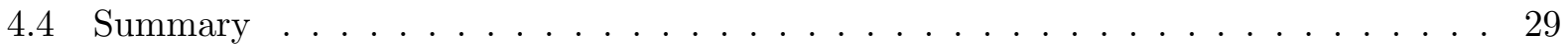

$\begin{array}{lll}5 & \text { Summary } & 31\end{array}$

6 References $\quad 32$ 


\section{FIGURES}

1 Schematics showing the path of multifrequency hops associated with the $\mathrm{Ag}_{\mathrm{Si}}-\mathrm{Va} \mathrm{Si}$ pair, including (a) vacancy-silver exchange hops, (b) vacancy rotation hops, (c) 2nd nearest-neighbor dissociation/association hops, (d) 3rd nearest-neighbor dissociation/association hops, (e) 4th nearest-neighbor dissociation/association hops. . . . . 3

2 Schematics showing the path of multifrequency hops associated with the $\mathrm{Ag}_{\mathrm{C}}-\mathrm{Va} \mathrm{C}_{\mathrm{C}}$ pair, including (a) vacancy-silver exchange hops, (b) vacancy rotation hops (two types), (c) 2nd nearest-neighbor dissociation/association hops, (d) 3rd nearest-neighbor dissociation/association hops (two types), (e) 4th nearest-neighbor dissociation/association hops. . . . . . . . . . . . . . . . . . . . . 4 4

3 Schematics showing the path of multifrequency hops associated with the $\mathrm{Ag}_{\mathrm{Si}}-\mathrm{Va}_{\mathrm{C}}$ pair, including (a) vacancy-silver exchange hops, (b) vacancy rotation hops, (c) 2nd nearest-neighbor dissociation/association hops, and (d) 3rd nearest-neighbor dissociation/association hops. . . . . . . . . . . . . . . . . 4

4 Schematics showing the path of multifrequency hops associated with the $\mathrm{Ag}_{\mathrm{C}}-\mathrm{Va} \mathrm{Si}_{\mathrm{Si}}$ pair, including (a) vacancy-silver exchange hops, (b) vacancy rotation hops, (c) 2nd nearest-neighbor dissociation/association hops, and (d) 3rd nearest-neighbor disso-

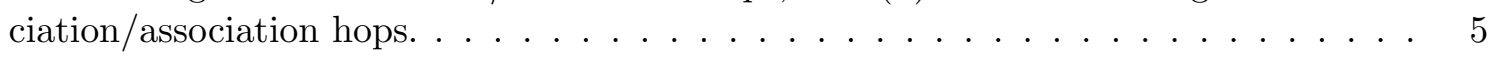

5 Plots showing the upper and lower bounds of the tracer diffusion coefficients of $\mathrm{Ag}$ and $\mathrm{C}$ in $\mathrm{SiC}$ as a function of $1 / \mathrm{T} \ldots \ldots \ldots \ldots \ldots$

6 Plots showing the partial diffusion coefficients of $\mathrm{Ag}$ and $\mathrm{C}$ as a function of $1 / \mathrm{T}$. . . 9

$7 \quad$ Plots showing the correlation factor of $\mathrm{Ag}$ diffusion in $\mathrm{SiC}$ as a function of $1 / \mathrm{T}$. . . 10

8 2-D and 3-D polycrystalline $\mathrm{SiC}$ microstructures considered in this study. The green, red, and yellow spheres represent lattice $\mathrm{Si} / \mathrm{C}$ atoms, GB Si/C atoms, and Ag atoms, respectively. . . . . . . . . . . . . . . . . . 13

9 Time-dependent mean squared displacements (MSD) of Ag atoms diffusing along GBs in 2-D and 3-D SiC polycrystals at various temperatures. The dashed lines represent linear fitting of MSD vs. time data. . . . . . . . . . . . . . . . 14

10 Arrhenius plot for $\mathrm{GB}$ diffusivity of $\mathrm{Ag}$ in $\mathrm{SiC}$. The solid line represents the best fitting of MD calculated data using the Arrhenius equation. . . . . . . . . . . . . 15

11 Formation energy of a $\mathrm{Ag}$ interstitial at various locations close to a GB in SiC. . . . 15

12 Time-dependent MSD of $\mathrm{Ag}$ atoms diffusing in bulk crystalline $\mathrm{SiC}$ at various tem-

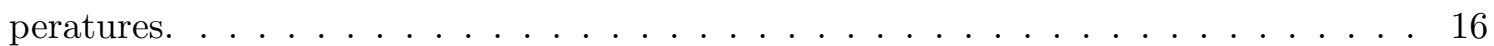

13 Snapshots of MD simulations of Ag interstitial diffusion in bulk crystalline $\mathrm{SiC}$ at $2500 \mathrm{~K}$. For easy visualization, only the silver atoms are shown as yellow spheres. . . 17

14 TAD simulation of $\mathrm{Ag}$ diffusion in a crystalline $\mathrm{SiC} \ldots \ldots \ldots \ldots$

15 TAD simulation of $\mathrm{Ag}$ diffusion in amorphous $\mathrm{SiC} \ldots \ldots \ldots \ldots$

16 Simple periodic microstructures with two grain boundaries (blue) (a) along the $x$ axis, and (b) intersecting. $L_{x}$ and $L_{y}$ are the domain lengths along the $x$ and $y$-axis, respectively. $w_{G B}$ is the GB width. $l_{G B, x}$ and $l_{G B, y}$ are the length of he grain boundaries along the $x$ and $y$-axis, respectively, and $l_{b, x}$ is the length of the grain along the $x$-axis. 
17 Comparison of the percentage errors in effective diffusion coefficients derived by different scaling approaches applied to the microstructure shown in Fig. 16a along the (a) $x$-axis and the (b) $y$-axis, and in Fig. 16b using the approach (c) $2 \mathrm{a}$ and (d) $2 \mathrm{~b}$. The 'no change' approach significantly overestimates $D^{e f f}$ in all directions. The 'constant product' approach increasingly underestimates $D_{1, x}^{\text {eff }}$ as $w_{G B}$ increases, and even more so for lower $D_{G B} / D_{b}$ values. Predictions for $D_{1, y}^{e f f}$ can also be as inaccurate as the 'no change' for large $D_{G B} / D_{b}$ values. The 'analytical' approaches, by definition, predict the same effective diffusion coefficient independently of $w_{G B}$ for the configurations it is based on. For the others, it performs better than the 'constant product' approach. The 'analytical 1x1y' approach consistently provides errors below $5 \%$ in the configurations studied. . . . . . . . . . . . . . .

18 Verification of the scale-up approaches on polycrystalline microstructures with continuous interfaces. $(\mathrm{a}, \mathrm{b}, \mathrm{c})$ show the error in the prediction of $D^{\text {eff }}$ for two different microstructures with different grain elongations for $D_{G B} / D_{b}=10$. (c) differs from (b) in the direction for which $D^{e f f}$ was derived, as shown by the arrow. (d,e,f) show the results for the same study, but with $D_{G B} / D_{b}=10^{3}$. The introduction of the continuous interfaces and the more complex microstructures lead to greater errors than in Fig. 17. However, the error remains lower than $30 \%$ for the cases studied for low $D_{G B} / D_{b}$ ratio (a,b,c). For higher $D_{G B} / D_{b}$ ratio $(\mathrm{d}, \mathrm{e}, \mathrm{f}), D^{\text {eff }}$ can be overestimated by $40 \%$ for equiaxed grains. Remarkably, the predictions are very accurate along elongated grain (e) which is the case of interest for $\mathrm{Ag}$ diffusion in $\mathrm{SiC}$. In all cases, each analytical approach provides similar predictions. Domain size is $1000 \times$

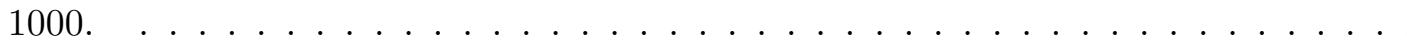

19 (a) Simulated grain dimensions (black dots) compared against the AGR-1 grain dimensions with twins included for all batches provided by Ref. [27]. The simulated microstructures cover the variations in grain dimensions found in the AGR-1 fuel batches. (b) and (c) show two examples of polycrystalline SiC microstructures created for this study, corresponding to the (b) bottom left and (c) top right points shown in (a). Domain size is $900 \mu \mathrm{m} \times 900 \mu \mathrm{m}$, and GBs are shown in blue and white. 25

20 Derivation of the effective Ag diffusion coefficient as a function of the temperature $T$, the grain minor axis length $m_{i}$, and the grain major axis length $m_{a}$. (a,b) show how $D 0$ depends on (a) $m_{i}$ and (b) $m_{a}$. It seems to slightly decrease with increasing $m_{i}$. (c,d) show how $Q$ depends on (c) $m_{i}$ and (d) $m_{a}$. While $Q$ seems independent of $m_{a}$, it increases linearly with the $m_{i}$. (e,f) show how the effective diffusion coefficient $D^{e f f}$ depends on (e) $m_{i}$ and (f) $m_{a}$ for different temperatures. The best fit using the average value for $D_{0}$ and the linear fit for $Q\left(m_{i}\right)$ was also added on (e,f). The fit successfully captures the effects of temperature and microstructure on $D^{\text {eff }}$. . . 27

21 Comparison of the Ag diffusion coefficients provided by atomistic simulations for the bulk $D_{\text {bulk }}$ and the GBs $D_{G B}$, the coefficient previously used in Bison $D_{B i s o n}$ provided in Ref. [1], and the effective diffusivity values provided by the current mesoscale study $D^{e f f}$. The different values for $D^{e f f}$ at each temperatures correspond to the extreme grain minor axis length values of the $\mathrm{SiC}$ microstructures described in Fig. 19. The effective Ag diffusion coefficient derived through atomistic and mesoscale calculations falls close to the empirical value currently used in Bison. Multiplying $D_{0}\left(m_{i}\right)$ by a corrective factor $\delta_{\text {corr }}=8.5$ is enough to increase the calculated effective Ag diffusivity to the same level than the empirical value used in Bison while still accounting for the effect of the microstructure, as shown by $D^{e f f, c o r r} \ldots \ldots \ldots \ldots$ 
22 Comparison of measured (PIE) and computed (PARFUME, Bison) silver release fractions for AGR-1 compacts. (a) shows the results with the current empirical diffusivity currently in Bison, (b) with the effective Ag diffusion coefficient derived from multiscale approach derived in this study (Eq. (19)), (c) with the corrected effective $\mathrm{Ag}$ diffusion coefficient (Eq. (20)). The underestimation of the Ag diffusivity in $\mathrm{SiC}$ noted in Fig. 21 leads to an underestimation of the $\mathrm{Ag}$ release fraction. However, using the corrected effective Ag diffusion coefficient provides better predictions that can be compared with the current Bison model. . . . . . . . . . . . . . 30 


\section{TABLES}

$1 \quad$ DFT calculation results of all migration barriers of hops described in Section 2.1.1 . 7

2 Different analytical approaches for scale up. . . . . . . . . . . . . . 22

3 Parameters used to compare the errors in $D^{\text {eff }}$ from the different scaling approaches in Fig. 16. . . . . . . . . . . . . . . . . . . . . . . . 22

4 Microstructure properties of AGR-1 fuel capsules. . . . . . . . . . . . . . 29 


\section{INTRODUCTION}

As one of the Generation IV advanced reactor designs, the Very High Temperature Reactor (VHTR) employs tristructural isotropic (TRISO) fuel to provide nuclear power. A TRISO particle contains a fuel kernel coated with a porous carbon buffer layer, an inner Pyrolytic Carbon (IPyC) layer, a silicon carbide $(\mathrm{SiC})$ layer, and an outer $\mathrm{PyC}(\mathrm{OPyC})$ layer [1]. While the $\mathrm{SiC}$ layer provides an effective barrier to prevent the release of fission products, several of them (e.g. silver $[\mathrm{Ag}]$ and cesium [Cs]), have been observed to escape from intact TRISO particles [2]. The release of silver is a major contamination concern due to the long half-life $(\approx 250$ days $)$ of the ${ }^{110 m} \mathrm{Ag}$ isotope.

To date, several mechanisms have been proposed to explain the Ag release: (i) Ag diffusion through the grain boundaries (GBs) of polycrystalline SiC microstructure [3, 4]; (ii) Ag transport assisted by the chemical attack of the $\mathrm{SiC}$ layer by $\mathrm{Pd}$ atoms, which transforms $\mathrm{SiC}$ into a mixture of palladium silicide and graphite [5,6]; (iii) void mediated transport of $\mathrm{Ag}$ [7]; (iv) diffusion of $\mathrm{Ag}$ vapor through nanocracks in $\mathrm{SiC}$ coatings [8,9]; and (v) neutron irradiation-enhanced $\mathrm{Ag}$ transport in $\mathrm{SiC}$ [10]. Despite nearly three decades of research, the dominant mechanism for $\mathrm{Ag}$ release is still not fully understood. Due to the low melting point of pure $\mathrm{Ag}\left(962{ }^{\circ} \mathrm{C}\right)$ and its high vapor pressure, it is also not feasible to directly measure $\mathrm{Ag}$ diffusivity in $\mathrm{SiC}$ using the traditional diffusion couple method. There is a further issue due to the essentially zero solubility of $\mathrm{Ag}$ in $\mathrm{SiC}$, which prevents interdiffusion between $\mathrm{SiC}$ and $\mathrm{Ag}$. In order to accumulate sufficiently high $\mathrm{Ag}$ concentration in $\mathrm{SiC}$ for diffusion profile analysis, $\mathrm{Ag}$ atoms have to be forced into the $\mathrm{SiC}$ lattice via ion implantation [8]. However, such a non-equilibrium process will introduce a significant amount of radiation damage (e.g., defect generation and amorphization) that will interact with implanted $\mathrm{Ag}$ atoms. Furthermore, as will be shown in Section 3.2, the injected Ag atoms in SiC lattice tend to aggregate with each other to form large Ag precipitates, which is a thermodynamically favorable process. Both factors may prevent a clear interpretation of the results from ion-implantation experiments.

For accurate prediction of Ag release from TRISO particles using the finite-element-based code Bison [1], it is critical to obtain the Ag diffusion coefficient through the SiC layer. The goal of this fiscal year 2021 NEAMS project is to use a multiscale modeling approach combining atomistic and phase-field methods to inform Bison of the effective Ag diffusivity in SiC. Experimentally, it has been observed that $\mathrm{Ag}$ accumulated in grain boundaries and triple junctions in $\mathrm{SiC}$ [3]. We therefore adopt the hypothesis that GB diffusion is the primary pathway for Ag penetration of the $\mathrm{SiC}$ layer. Instead of using bulk amorphous $\mathrm{SiC}$ as a structural model for $\mathrm{SiC} \mathrm{GB}$ [4], direct molecular dynamics simulations of Ag atoms diffusing along GBs in SiC polycrystals have been performed in this study. For comparison, diffusion of both substitutional and interstitial Ag defects through bulk SiC have also been studied.

This report is organized as follows. In Section 2, the diffusivity of $\mathrm{Ag}$ in bulk $\mathrm{SiC}$ has been determined using a combination of density functional theory, nudged elastic band method, and kinetic Monte-Carlo simulations. In Section 3, the diffusivity of Ag along grain boundaries in $\mathrm{SiC}$ has been obtained using molecular dynamics simulations. Finally, in Section 4, the effective diffusion coefficient of $\mathrm{Ag}$ through a polycrystalline $\mathrm{SiC}$ microstructure containing a network of grain boundaries has been calculated using mesoscale simulations. Ag release calculations have also been performed using Bison and the results are compared to available experimental data. 


\section{ATOMISTIC MODELING OF FISSION PRODUCT DIFFUSION IN BULK SiC}

Diffusion of $\mathrm{Ag}$ in bulk $\mathrm{SiC}$ is one of the potential pathways for fission product penetration in the $\mathrm{SiC}$ layer. It also allows $\mathrm{Ag}$ atoms to move to $\mathrm{SiC}$ grain boundaries as a fast diffusion pathway. In this section, the tracer diffusion coefficient of the fission product $\mathrm{Ag}$ in bulk $\mathrm{SiC}$ is investigated by density functional theory (DFT) and kinetic Monte Carlo (kMC) simulation. Despite the fact Ag diffusion in $\mathrm{SiC}$ has been studied by experiments and atomistic simulations, there are discrepancies between modeling and experiments [11]. The previous atomistic simulation studies include the work on $\mathrm{Ag}$ diffusion along $\Sigma 3$ grain boundaries by Khalil et al.[12], Ag transport in high-energy grain boundaries by Ko et al.[4], as well as diffusion in the bulk SiC by Shrader et al.[13]. Note that while the diffusion of $\mathrm{Ag}$ in bulk $\mathrm{SiC}$ has been investigated by DFT, it has never been applied to quantify the cumulative effect of a wide variety of symmetrically nonequivalent hops, especially for vacancy-mediated substitutional diffusion. The substitutional diffusion in Ag is strongly associated with different types of vacancy-defect pairs and their binding energies. In this work, the cumulative effect of multifrequency diffusion hops is investigated by building a rigorous diffusion model that accounts for all essential hops. The migration barrier associated with each hop is then determined by DFT with the nudged elastic band (NEB) method. The tracer diffusion coefficient of Ag is evaluated by $\mathrm{kMC}$ based on the NEB data. Note that only the neutral charge state is considered in the scope of this work.

\subsection{Methods}

\subsubsection{Multifrequency diffusion model}

A total of 24 symmetrically nonequivalent vacancy-atom hops are considered in the study of $\mathrm{Ag}$ substitutional diffusion in the single-crystal SiC. It is assumed that $\mathrm{Ag}$ can affect the migration barriers of atom-vacancy exchanges associated with the atom (either $\mathrm{Si}$ or $\mathrm{C}$ ) at the first-nearest neighbor of the $\mathrm{Ag}$ atom, and the hops that happen farther from $\mathrm{Ag}$ than the first-nearest neighbor shell are treated the same as the substitutional diffusion of single-crystal SiC. The work also reasons that the diffusion paths causing the formation of anti-site defects are energetically unfavorable, and thus their contributions to Ag diffusion can be considered negligible. This multifrequency approach is similar to the previous study by Cooper et al. [14] using a simplified five-frequency model. In this work we identified the symmetrically nonequivalent diffusion paths in a more rigorous manner and further examined the hops associated with the following $\mathrm{Ag}$-vacancy pairs: $\mathrm{Ag}_{\mathrm{Si}}-\mathrm{Va}_{\mathrm{Si}}, \mathrm{Ag}_{\mathrm{C}}-\mathrm{Va}_{\mathrm{C}}$, $\mathrm{Ag}_{\mathrm{Si}}-\mathrm{Va}_{\mathrm{C}}$, and $\mathrm{Ag}_{\mathrm{C}}-\mathrm{Va}_{\mathrm{Si}}$.

For the hopping paths associated with the $\mathrm{Ag}_{\mathrm{Si}}-\mathrm{Va}_{\mathrm{Si}}$ pair, the multifrequency hops of $\mathrm{Si}$ are similar to those of the five-frequency model for face-centred cubic (fcc) system. The hops of vacancyAg exchange, vacancy-Ag rotation, and dissociation/association are summarized by the schematics in Fig. 1 showing the seven symmetrically nonequivalent hopping paths on the (111) lattice plane of $\mathrm{SiC}$. Note that each line in Fig. 1 represents the hopping path with an atom ( $\mathrm{Ag}$ or $\mathrm{Si}$ ) at one end and a vacant $\mathrm{Si}$ site at the other end. Fig. 1(a) shows the exchange hops between $\mathrm{Ag}_{\mathrm{Si}}$ and $\mathrm{Va}_{\mathrm{Si}}$. The rotation hops associated with the $\mathrm{Ag}_{\mathrm{Si}}-\mathrm{Va}_{\mathrm{Si}}$ pair consist of two unique paths. For the first (second) types of rotation hops, the nearest $\mathrm{C}$ atom shared with the moving Si atom and vacancy is the first (second) nearest neighbor $\mathrm{C}$ atom with respect to Ag. The two different rotation paths of the first and second types of rotation are shown in green and pink lines in Fig. 1(b), respectively. Since the hopping path of the type-I rotation hops may cause more displacement of the carbon 

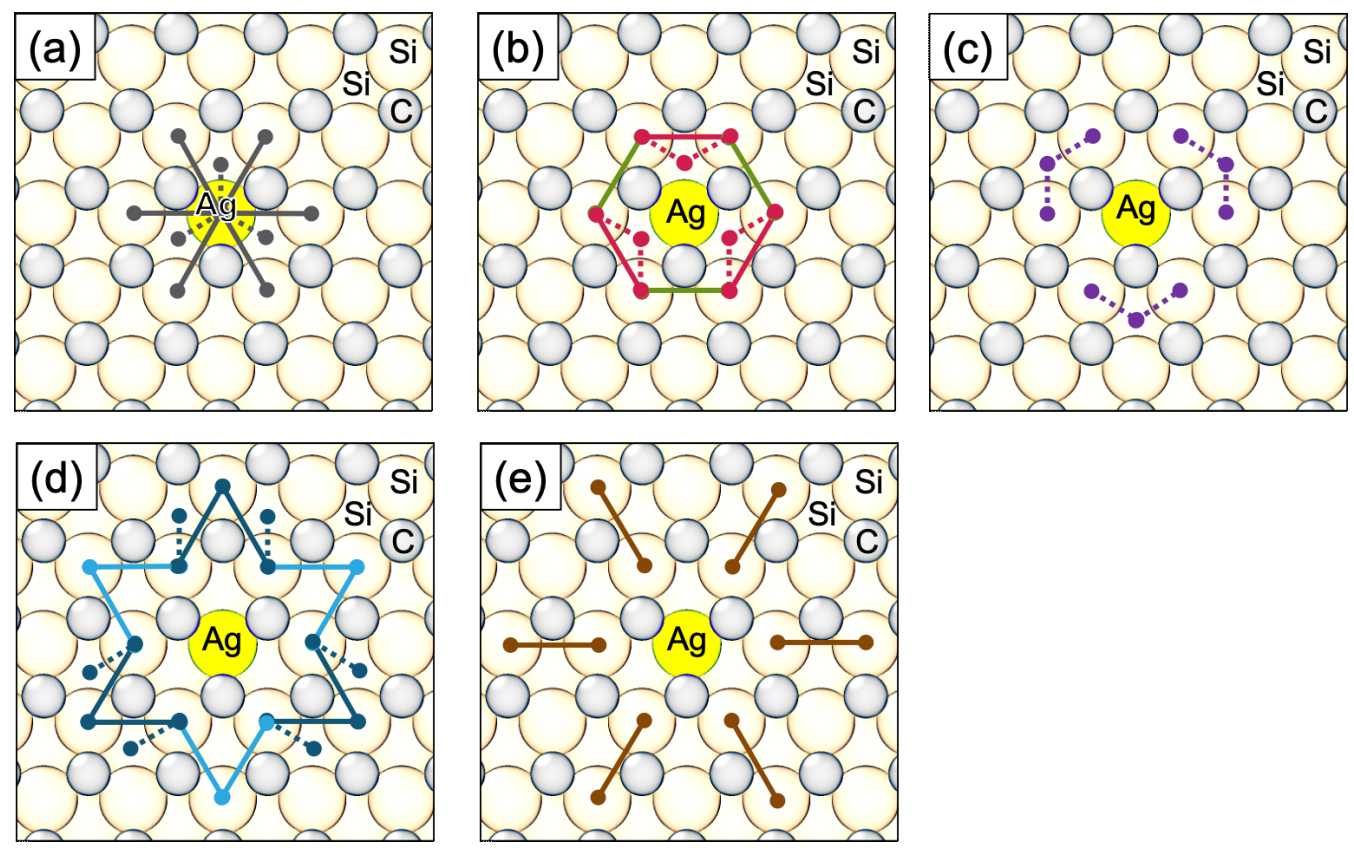

Figure 1: Schematics showing the path of multifrequency hops associated with the $\mathrm{Ag}_{\mathrm{Si}}-\mathrm{Va}_{\mathrm{Si}}$ pair, including (a) vacancy-silver exchange hops, (b) vacancy rotation hops, (c) 2nd nearest-neighbor dissociation/association hops, (d) 3rd nearest-neighbor dissociation/association hops, (e) 4th nearestneighbor dissociation/association hops.

atom (or local distortion) which is at the center of the 3Si-1Ag tetrahedron, the migration barrier of the type-I rotation hop is expected to be higher than that of the type-II rotation hop.

The vacancy-silver dissociation/association hops from/to the 2nd, 3rd, and 4th nearest-neighbor positions with respect to the $\mathrm{Ag}$ atom are shown in Fig. 1(c), (d), and (e), respectively. For each of the 2nd and 4th dissociation/association hops, there is one symmetrically nonequivalent hop in each case, which is similar to the five-frequency model in fcc system. The 2nd and 4th dissociation/association hops are shown in Fig. 1(c) and (e), respectively. Note there are two different 3rd dissociation/association hops. For the first (second) type of 3rd dissociation/association hops, the nearest $\mathrm{C}$ atom shared with the moving $\mathrm{Si}$ atom and vacancy is the $2 \mathrm{nd}$ (3rd) nearest neighbor $\mathrm{C}$ atom with respect to Ag. The first and second types of 3rd dissociation/association hops are displayed by the dark blue and light blue lines in Figure 1 (d), respectively.

The hopping paths associated with the $\mathrm{Ag}_{\mathrm{C}}-\mathrm{Va}_{\mathrm{C}}$ pair can also be determined in the same way considering the multifrequency hops of $\mathrm{C}$ and $\mathrm{Ag}_{\mathrm{C}}$, including one vacancy-Ag exchange, two vacancy rotation hops, and four vacancy dissociation/association hops. The seven symmetrically nonequivalent hops are illustrated in Fig. 2.

The vacancy-atom hops associated with the $\mathrm{Ag}_{\mathrm{Si}}-\mathrm{Va}_{\mathrm{C}}$ pair consist of four symmetrically nonequivalent paths. Fig. 3(a)-(d) illustrates the hops of vacancy-Ag exchange, vacancy rotation, and 2nd and 3rd nearest-neighbor dissociation/association, respectively. Note that unlike $\mathrm{Ag}_{\mathrm{C}}-\mathrm{Va}_{\mathrm{C}}$ and $\mathrm{Ag}_{\mathrm{Si}}-\mathrm{Va}_{\mathrm{Si}}$ pairs, there is only a single type of rotation hop. The vacancy-atom hops associated with the $\mathrm{Ag}_{\mathrm{C}}-\mathrm{Va}$ Si pair can also be described accordingly, as illustrated in Fig. 4 (a)-(d). 

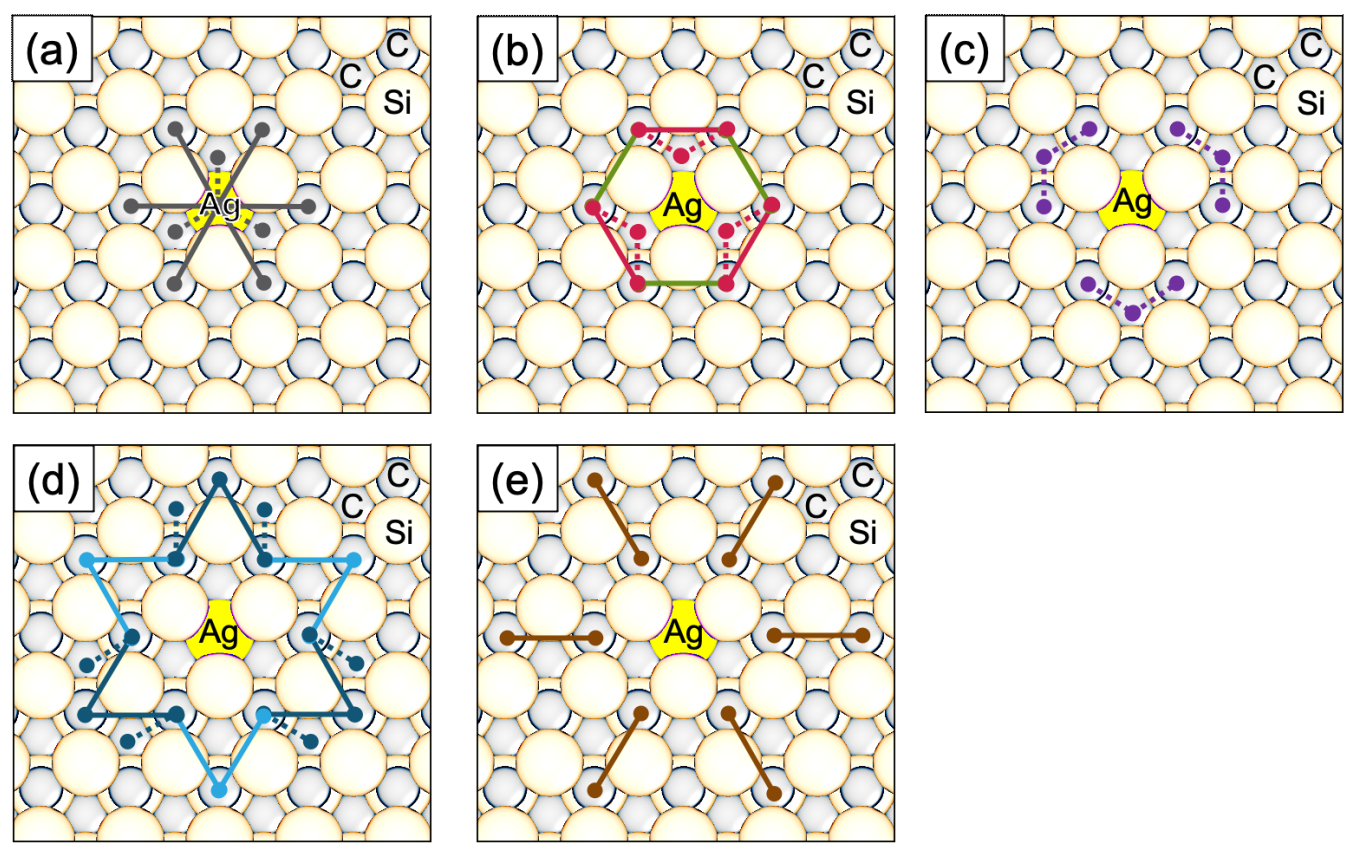

Figure 2: Schematics showing the path of multifrequency hops associated with the $\mathrm{Ag}_{\mathrm{C}}-\mathrm{Va}_{\mathrm{C}}$ pair, including (a) vacancy-silver exchange hops, (b) vacancy rotation hops (two types), (c) 2nd nearestneighbor dissociation/association hops, (d) 3rd nearest-neighbor dissociation/association hops (two types), (e) 4th nearest-neighbor dissociation/association hops.
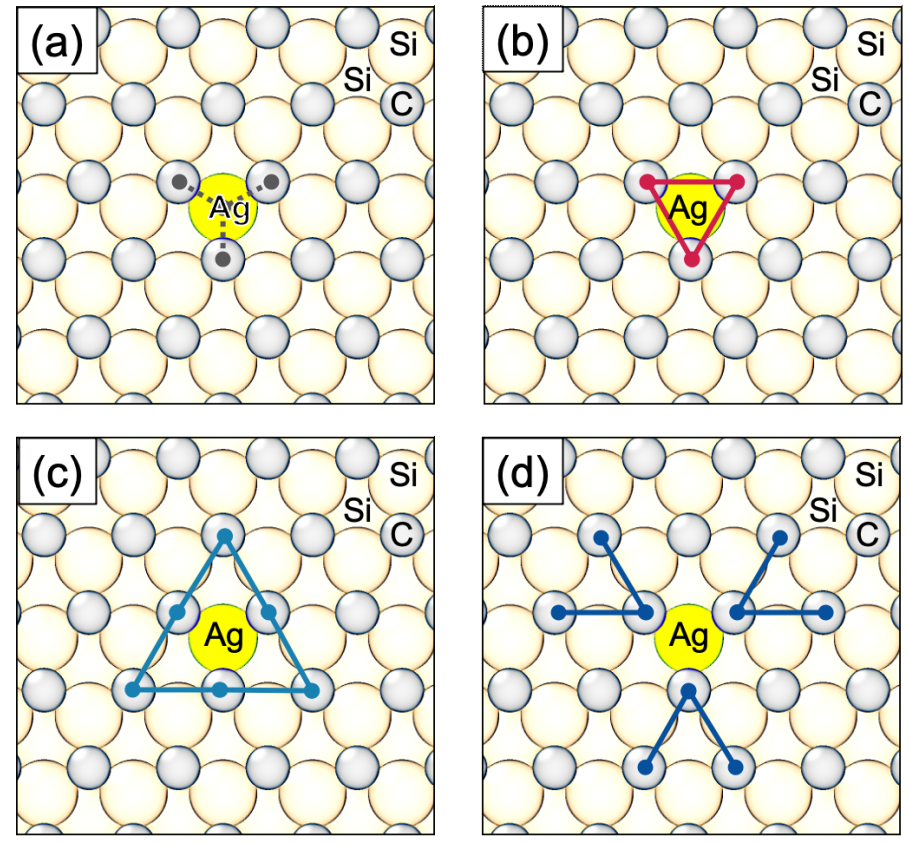

Figure 3: Schematics showing the path of multifrequency hops associated with the $\mathrm{Ag}_{\mathrm{Si}}-\mathrm{Va}_{\mathrm{C}}$ pair, including (a) vacancy-silver exchange hops, (b) vacancy rotation hops, (c) 2nd nearest-neighbor dissociation/association hops, and (d) 3rd nearest-neighbor dissociation/association hops. 

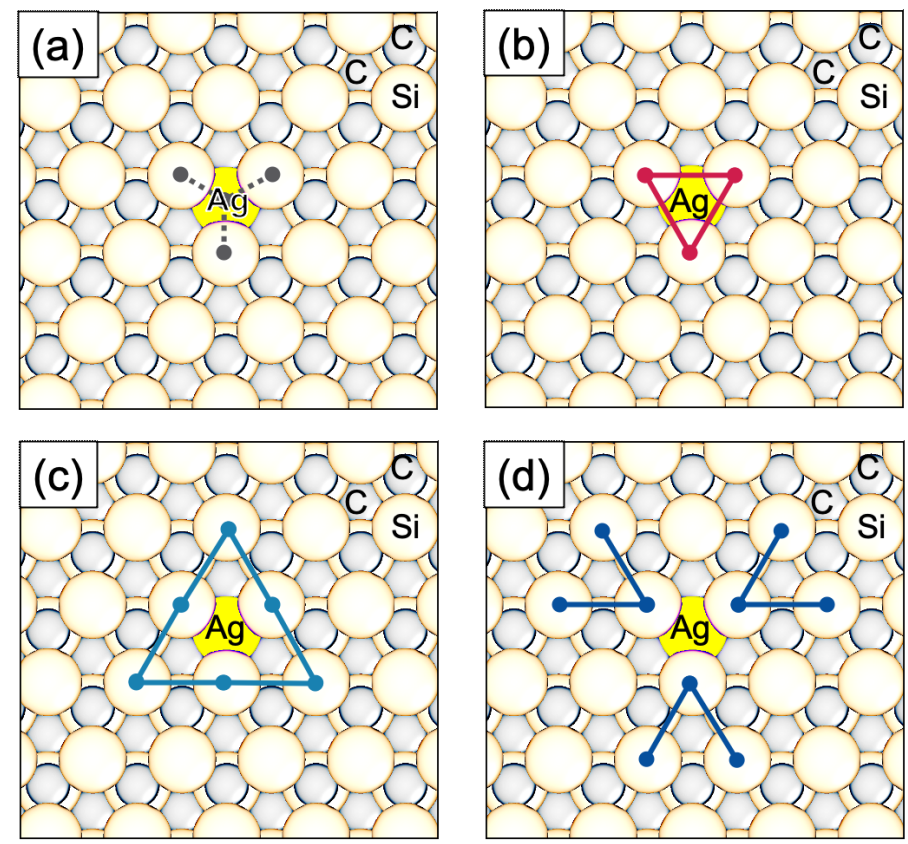

Figure 4: Schematics showing the path of multifrequency hops associated with the $\mathrm{Ag}_{\mathrm{C}}-\mathrm{Va}_{\mathrm{Si}}$ pair, including (a) vacancy-silver exchange hops, (b) vacancy rotation hops, (c) 2nd nearest-neighbor dissociation/association hops, and (d) 3rd nearest-neighbor dissociation/association hops.

\subsubsection{First-Principles Methodologies}

The ground state energies and migration barriers are calculated using the Vienna Ab initio Simulation Package (VASP). All calculations were spin-polarized and initialized under the ferromagnetic ordering. The plane wave energy cutoff was selected to be $600 \mathrm{eV}$ for all calculations. The interactions between ions and core electrons are described using the projector augmented wave (PAW) method. The Perdew-Burke-Ernzerhof parameterization of the generalized gradient approximation was used for the exchange correlation potentials. The Brillouin zone was sampled using a $4 \times 4 \times 4 \mathrm{k}$-point mesh generated using the Monkhorst-Pack scheme. Calculations were performed with a 216 -site periodic simulation cell, which is a $3 \times 3 \times 3$ supercell of the zinc blende (sphalerite) unit cells of $\mathrm{SiC}$. The transition state and migration barrier were determined by climbing-image nudged-elastic-band (CI-NEB) calculations [15]. The Quick-Min force-based optimizer was used to determine the minimum energy paths and saddle points, and the optimizer was implemented by the Transition State Tools for VASP (VTST). In all ground-state DFT and CI-NEB runs, the break condition of energy convergence for electronic relaxation is $10^{-6} \mathrm{eV}$ and force for ionic relaxation is $0.01 \mathrm{eV} / \AA$.

\subsubsection{Kinetic Monte Carlo Methods}

The kMC simulations were carried out using the migration barriers derived by DFT. The kMC model assumes atomic hops beyond the range of the hops listed in Table 1 will be the same as the diffusion events in $\mathrm{SiC}$ without $\mathrm{Ag}$. The $\mathrm{kMC}$ model implemented the kinetically resolved activation (KRA) barriers to determine the hopping events. The KRA barriers were calculated from the ground-state and transition-state energies. The attempt frequencies of all vacancy-mediated hops are assumed to be $5 \times 10^{12} \mathrm{~Hz}$. The $20 \times 20 \times 20$ supercell of the primitive unit cell was used 
with a total of 16,000 atomic sites, including $1 \mathrm{Ag}$ atom and 1 carbon vacancy in a $\mathrm{SiC}$ background supercell. Each kMC run was initiated with 100 passes for configuration equilibration, followed by 10,000 passes to determine the diffusion coefficient, which was sampled in a period of 10 passes. KMC runs were performed for temperatures from 1073 to $2073 \mathrm{~K}$.

\subsection{Density Functional Theory Calculation Results}

Table 1 shows the migration barriers of all symmetrically-unique hops calculated by using DFT and CI-NEB methods. The hops include those without Ag and four types of Ag-Va pairs. The calculated migration barriers of $\mathrm{C}$ and $\mathrm{Si}$ in $\mathrm{SiC}$ without $\mathrm{Ag}$ are 3.51 and $3.59 \mathrm{eV}$, respectively, which are in good agreement with early atomistic simulation studies [16, 17]. The migration barriers of all Ag-vacancy exchanges are all significantly lower than most of the hops involving $\mathrm{C}$ or $\mathrm{Si}$ as the moving specie. Note that for $\mathrm{Ag}_{\mathrm{Si}}-\mathrm{Va}_{\mathrm{C}}$ and $\mathrm{Ag}_{\mathrm{C}}-\mathrm{Va}$ Si pairs, the exchange hop of $\mathrm{Ag}$ and vacancy is a migration with zero energy barrier, as the ground-state equilibrium configurations and energies of the two endpoints are equivalent. These equivalent endpoint configurations and energies also happen for the $2 \mathrm{nn}$ dissociation/association hop with respect to the $\mathrm{Ag}_{\mathrm{C}}-\mathrm{Va}_{\mathrm{Si}}$ pair, as marked by the symbol $*$ in Table 1. For $\mathrm{Ag}_{\mathrm{C}}-\mathrm{Va}_{\mathrm{C}}$ and $\mathrm{Ag}_{\mathrm{Si}}-\mathrm{Va}_{\mathrm{Si}}$ pairs, the migration barriers of the type-I rotation hop are both higher than the type-II rotation hop. This indicates that specific pathways are preferred for Ag-vacancy pairs to move together, and the correlation factor of Ag diffusion will differ from the analytical expression compared to the five-frequency model applied in the study [14].

The DFT calculation results in Table 1 also suggest medium to strong binding of the four types of Ag-vacancy pairs. The range of binding energy for $\mathrm{Ag}_{\mathrm{Si}}-\mathrm{Va}_{\mathrm{Si}}, \mathrm{Ag}_{\mathrm{C}}-\mathrm{Va}_{\mathrm{C}}, \mathrm{Ag}_{\mathrm{Si}}-\mathrm{Va}_{\mathrm{C}}$, and $\mathrm{Ag}_{\mathrm{C}}-\mathrm{Va}_{\mathrm{Si}}$ are respectively 0.3-0.7, 2.2-2.8, 3.0-3.6, and $\sim 6.9 \mathrm{eV} /$ atom. It can be found that the Ag-vacancy pairs involving different sublattice sites of $\mathrm{SiC}$ have much stronger binding than those involving the same sublattice sites. The strong binding of $\mathrm{Ag}_{\mathrm{Si}}-\mathrm{Va}_{\mathrm{C}}$ and $\mathrm{Ag}_{\mathrm{C}}-\mathrm{Va}_{\mathrm{Si}}$ pairs makes them an effective trapping site for $\mathrm{Ag}$, and because of their extremely high migration barriers of rotation hops (7.31 and $8.05 \mathrm{eV}$ ), the $\mathrm{Ag}$ becomes immobilized whenever it binds with the vacancy at the different sublattice site from that of Ag. For the vacancy that occupies the same sublattice site as Ag, the migration barrier of rotation hops are significantly lower than the previous cases, so the Ag-vacancy pair of the same sublattice site still remains mobile at the temperature of interest for the TRISO fuel.

Note that the difference in thermodynamic stability and mobility of these Ag-vacancy pairs will make the Ag transport in irradiated environments more complicated than in equilibrium conditions. At thermodynamic equilibrium, the Ag transport is determined by the formation energy of defects and their mobility. In this work, the $\mathrm{Va}_{\mathrm{C}}$ formation energy is calculated to be between $4.18 \mathrm{eV}$ (Si-rich) and $4.72 \mathrm{eV}$ (C-rich), and the Vasi formation energy is between $7.17 \mathrm{eV}$ (C-rich) and $7.72 \mathrm{eV}$ (Si-rich). The formation energy of interstitial Ag tetragonally coordinated between four C atoms $\left(\mathrm{Ag}_{\mathrm{TC}}\right)$ is calculated as $10.25 \mathrm{eV}$. It is suggested that the substitutional diffusion mediated by carbon vacancies will be the dominant diffusion path under thermodynamic equilibrium. While the migration barrier of $\mathrm{Ag}_{\mathrm{TC}}$ interstitials is calculated as low as $1.01 \mathrm{eV}$, the high formation energy $(>10 \mathrm{eV})$ suggests that $\mathrm{Ag}_{\mathrm{TC}}$ is not energetically favorable to form.

For the TRISO fuel under the service condition, the dominant Ag transport mechanism will depend on the concentration of all types of point defects generated by radiation damage, as well as their interactions with Ag. There are six types of point defects that can be produced by irradiation, including carbon vacancy, silicon vacancy, carbon antisite, silicon antisite, silver interstitial, carbon interstitial, and silicon interstitial. The relative concentrations of these point defects and Ag are strongly associated with the service conditions and microstructure, including neutron displacement 
Table 1: DFT calculation results of all migration barriers of hops described in Section 2.1.1

\begin{tabular}{|c|c|c|}
\hline Description of hops & Moving specie & Barrier $(\mathrm{eV})$ \\
\hline \multicolumn{3}{|l|}{ No Ag } \\
\hline $\mathrm{Va}_{\mathrm{Si}}$ exchange with $\mathrm{Si}_{\mathrm{Si}}$ & $\mathrm{Si}$ & 3.59 \\
\hline Va $_{\mathrm{C}}$ exchange with $\mathrm{C}_{\mathrm{C}}$ & $\mathrm{C}$ & 3.51 \\
\hline \multicolumn{3}{|l|}{$\mathrm{Ag}_{\mathrm{Si}}-\mathrm{Va}_{\mathrm{Si}}$ pair } \\
\hline $\mathrm{Va}_{\mathrm{Si}}$ exchange with $\mathrm{Ag}_{\mathrm{Si}}(\mathrm{I})$ & $\mathrm{Ag}$ & 1.30 \\
\hline $\mathrm{Va}_{\mathrm{Si}}$ rotate $\mathrm{Ag}_{\mathrm{Si}}(\mathrm{I})$ & $\mathrm{Si}$ & 3.82 \\
\hline $\mathrm{Va}_{\mathrm{Si}}$ rotate $\mathrm{Ag}_{\mathrm{Si}}(\mathrm{II})$ & $\mathrm{Si}$ & 3.38 \\
\hline Va $_{S i}$ dissociate from $\mathrm{Ag}_{\mathrm{Si}}, 2 \mathrm{nn}$ & $\mathrm{Si}$ & 3.79 \\
\hline $\mathrm{Va}_{\mathrm{Si}}$ associate to $\mathrm{Ag}_{\mathrm{Si}}, 2 \mathrm{nn}$ & $\mathrm{Si}$ & 3.10 \\
\hline $\mathrm{Va}_{\mathrm{Si}}$ dissociate from $\mathrm{Ag}_{\mathrm{Si}}, 3 \mathrm{nn}(\mathrm{I})$ & $\mathrm{Si}$ & 3.84 \\
\hline $\mathrm{Va}_{\mathrm{Si}}$ associate to $\mathrm{Ag}_{\mathrm{Si}}, 3 \mathrm{nn}(\mathrm{I})$ & $\mathrm{Si}$ & 3.18 \\
\hline $\mathrm{Va}_{\mathrm{Si}}$ dissociate from $\mathrm{Ag}_{\mathrm{Si}}, 3 \mathrm{nn}$ (II) & $\mathrm{Si}$ & 3.54 \\
\hline $\mathrm{Va}_{\mathrm{Si}}$ associate to $\mathrm{Ag}_{\mathrm{Si}}, 3 \mathrm{nn}(\mathrm{II})$ & $\mathrm{Si}$ & 3.04 \\
\hline $\mathrm{Va}_{\mathrm{Si}}$ dissociate from $\mathrm{Ag}_{\mathrm{Si}}, 4 \mathrm{nn}$ & $\mathrm{Si}$ & 2.41 \\
\hline $\mathrm{Va}_{\mathrm{Si}}$ associate to $\mathrm{Ag}_{\mathrm{Si}}, 4 \mathrm{nn}$ & $\mathrm{Si}$ & 2.15 \\
\hline \multicolumn{3}{|l|}{$\mathrm{Ag}_{\mathrm{C}}-\mathrm{Va}_{\mathrm{C}}$ pair } \\
\hline $\mathrm{Va}_{\mathrm{C}}$ exchange with $\mathrm{Ag}_{\mathrm{C}}$ & $\mathrm{Ag}$ & 2.27 \\
\hline $\mathrm{Va}_{\mathrm{C}}$ rotate $\mathrm{Ag}_{\mathrm{C}}(\mathrm{I})$ & $\mathrm{C}$ & 4.38 \\
\hline $\operatorname{Va}_{\mathrm{C}}$ rotate $\operatorname{Ag}_{\mathrm{C}}(\mathrm{II})$ & $\mathrm{C}$ & 3.26 \\
\hline $\mathrm{Va}_{\mathrm{C}}$ dissociate from $\mathrm{Ag}_{\mathrm{C}}, 2 \mathrm{nn}$ & $\mathrm{C}$ & 4.90 \\
\hline $\operatorname{Va}_{\mathrm{C}}$ associate to $\mathrm{Ag}_{\mathrm{C}}, 2 \mathrm{nn}$ & $\mathrm{C}$ & 2.06 \\
\hline $\mathrm{Va}_{\mathrm{C}}$ dissociate from $\mathrm{Ag}_{\mathrm{C}}, 3 \mathrm{nn}(\mathrm{I})$ & $\mathrm{C}$ & 4.51 \\
\hline $\mathrm{Va}_{\mathrm{C}}$ associate to $\mathrm{Ag}_{\mathrm{C}}, 3 \mathrm{nn}(\mathrm{I})$ & $\mathrm{C}$ & 1.87 \\
\hline $\mathrm{Va}_{\mathrm{C}}$ dissociate from $\mathrm{Ag}_{\mathrm{C}}, 3 \mathrm{nn}$ (II) & $\mathrm{C}$ & 4.40 \\
\hline $\mathrm{Va}_{\mathrm{C}}$ associate to $\mathrm{Ag}_{\mathrm{C}}, 3 \mathrm{nn}(\mathrm{II})$ & $\mathrm{C}$ & 2.20 \\
\hline $\mathrm{Va}_{\mathrm{C}}$ dissociate from $\mathrm{Ag}_{\mathrm{C}}, 4 \mathrm{nn}$ & $\mathrm{C}$ & 4.26 \\
\hline $\mathrm{Va}_{\mathrm{C}}$ associate to $\mathrm{Ag}_{\mathrm{C}}, 4 \mathrm{nn}$ & $\mathrm{C}$ & 1.88 \\
\hline \multicolumn{3}{|l|}{$\mathrm{Ag}_{\mathrm{Si}}-\mathrm{Va}_{\mathbf{C}}$ pair } \\
\hline $\mathrm{Va}_{\mathrm{C}}$ exchange with $\mathrm{Ag}_{\mathrm{C}}$ & $\mathrm{Ag}$ & $0^{*}$ \\
\hline Vac rotate $A g_{C}$ & $\mathrm{C}$ & 7.31 \\
\hline $\mathrm{Va}_{\mathrm{C}}$ dissociate from $\mathrm{Ag}_{\mathrm{C}}, 2 \mathrm{nn}$ & $\mathrm{C}$ & 6.02 \\
\hline $\mathrm{Va}_{\mathrm{C}}$ associate to $\mathrm{Ag}_{\mathrm{C}}, 2 \mathrm{nn}$ & $\mathrm{C}$ & 2.45 \\
\hline $\mathrm{Va}_{\mathrm{C}}$ dissociate from $\mathrm{Ag}_{\mathrm{C}}, 3 \mathrm{nn}$ & $\mathrm{C}$ & 5.70 \\
\hline $\mathrm{Va}_{\mathrm{C}}$ associate to $\mathrm{Ag}_{\mathrm{C}}, 3 \mathrm{nn}$ & $\mathrm{C}$ & 2.66 \\
\hline \multicolumn{3}{|l|}{$\mathrm{Ag}_{\mathrm{C}}-\mathbf{V a}_{\mathrm{Si}}$ pair } \\
\hline $\mathrm{Va}_{\mathrm{Si}}$ exchange with $\mathrm{Ag}_{\mathrm{C}}$ & $\mathrm{Ag}$ & $0^{*}$ \\
\hline $\mathrm{Va}_{\mathrm{Si}}$ rotate $\mathrm{Ag}_{\mathrm{C}}$ & $\mathrm{Si}$ & 8.05 \\
\hline $\mathrm{Va}_{\mathrm{Si}}$ dissociate from $\mathrm{Ag}_{\mathrm{C}}, 2 \mathrm{nn}$ & $\mathrm{Si}$ & $0^{*}$ \\
\hline $\mathrm{Va}_{\mathrm{Si}}$ associate to $\mathrm{Ag}_{\mathrm{C}}, 2 \mathrm{nn}$ & $\mathrm{Si}$ & $0^{*}$ \\
\hline $\mathrm{Va}_{\mathrm{Si}}$ dissociate from $\mathrm{Ag}_{\mathrm{C}}, 3 \mathrm{nn}$ & $\mathrm{Si}$ & 11.41 \\
\hline $\mathrm{Va}_{\mathrm{Si}}$ associate to $\mathrm{Ag}_{\mathrm{C}}, 3 \mathrm{nn}$ & $\mathrm{Si}$ & 4.55 \\
\hline
\end{tabular}


damage rate, displacement efficiency, defect clustering, sink strength, and reactions among various types of defects, including point defects and extended defects. This information is currently uncertain, unknown, or unexplored for the TRISO fuel. Although the defect concentration and distribution in irradiated $\mathrm{SiC}$ are out of the scope of the current work, they can be considered in a rigorous manner in the future to enhance the model developed in this section. The current work can be enhanced by developing DFT- or MD-informed mesoscale models characterizing the time evolution of each type of defect under an irradiated environment.

\subsection{Kinetic Monte Carlo Simulation Results}

By using the diffusion model presented in Section 2.1.1 and migration barriers calculated by DFT (Table 1), kMC simulations were performed to investigate the cumulative effect of multifrequency hops. From early molecular dynamics simulation of displacement cascades in $\mathrm{SiC}$, carbon vacancy has been consistently observed as the dominant type of vacancy defect surviving displacement cascade [18]. The excessive carbon vacancies are able to pin $A g_{S i}$ due to the strong binding, high rotation and dissociation barriers, while $\mathrm{Ag}_{\mathrm{C}}$ remains mobile. Note that carbon vacancy is also the dominant diffusion pathway for Ag transport under the equilibrium condition, which might be achieved at high temperature due to the fast transport of defects to sinks. Therefore, the kMC simulation in this work focuses on the Ag transport mediated by carbon vacancies.

Fig. 5 shows the tracer diffusion coefficients of $\mathrm{Ag}$ and $\mathrm{C}$ in $\mathrm{SiC}$ mediated by the carbon vacancy. The lower and upper bounds are due to the range of the formation energy of carbon vacancy and its binding energy with $\mathrm{Ag}_{\mathrm{C}}$. The activation energy of the $\mathrm{Ag}$ tracer diffusion coefficient is calculated as between 4.76 and $5.91 \mathrm{eV}$. Fig. 6 shows the partial diffusion coefficients of $\mathrm{Ag}$ and $\mathrm{C}$ in SiC. The partial diffusion coefficient is related to the tracer diffusion coefficient by $D^{*} / X_{\mathrm{V}}$ where $X_{\mathrm{V}}$ is the equilibrium vacancy concentration. Note that $\mathrm{Ag}_{\mathrm{C}}$ is shown to be significantly more mobile than carbon due to the strong binding between $\mathrm{Ag}_{\mathrm{C}}$ and $\mathrm{V}_{\mathrm{C}}$ and the low-energy barrier of Ag-vacancy exchange, which also makes the Ag diffusion highly correlated with jump frequencies deviating from the probabilities calculated by the theory of random walk. Fig. 7 shows the correlation factor of $\mathrm{Ag}$ diffusion in $\mathrm{SiC}$. The correlation factor is significantly less than 1 at temperatures from $1073 \mathrm{~K}$ to $2073 \mathrm{~K}$, indicating there are many dependent hopping events of Ag that will not contribute to long-range transport.

\subsection{Summary}

In this section, density function theory and kinetic Monte Carlo simulations were used to study the diffusion of $\mathrm{Ag}$ in bulk SiC. A multifrequency diffusion model was built to account for a total of 24 symmetrically nonequivalent hops that involve the atom in the vicinity of the Ag atom. CI-NEB calculations were performed to derive the migration barrier of each hop. The results show that the Ag-vacancy pairs involving different sublattice sites of $\mathrm{SiC}$ will have much stronger binding than those involving the same sublattice sites. This characteristic will have significant influence in irradiated $\mathrm{SiC}$. The carbon vacancy is known to be the dominant vacancy-type point defect in $\mathrm{SiC}$ under irradiation, and thus the excessive carbon vacancy is able to trap and pin $\mathrm{Ag}_{\mathrm{Si}}$, while $\mathrm{Ag}_{\mathrm{C}}$ remains mobile. The tracer diffusion coefficient of $\mathrm{Ag}_{\mathrm{C}}$ under thermodynamic equilibrium and its partial diffusion coefficient are determined by kMC simulations. The Arrhenius equation describing the lower and upper bounds of the $\mathrm{Ag}_{\mathrm{C}}$ diffusion coefficient were derived as

$$
D_{\mathrm{Ag}}^{*}=2.4 \times 10^{-4} \exp \left(-\frac{5.34 \pm 0.58(\mathrm{eV})}{k_{B} T}\right)\left(\mathrm{m}^{2} / \mathrm{s}\right)
$$




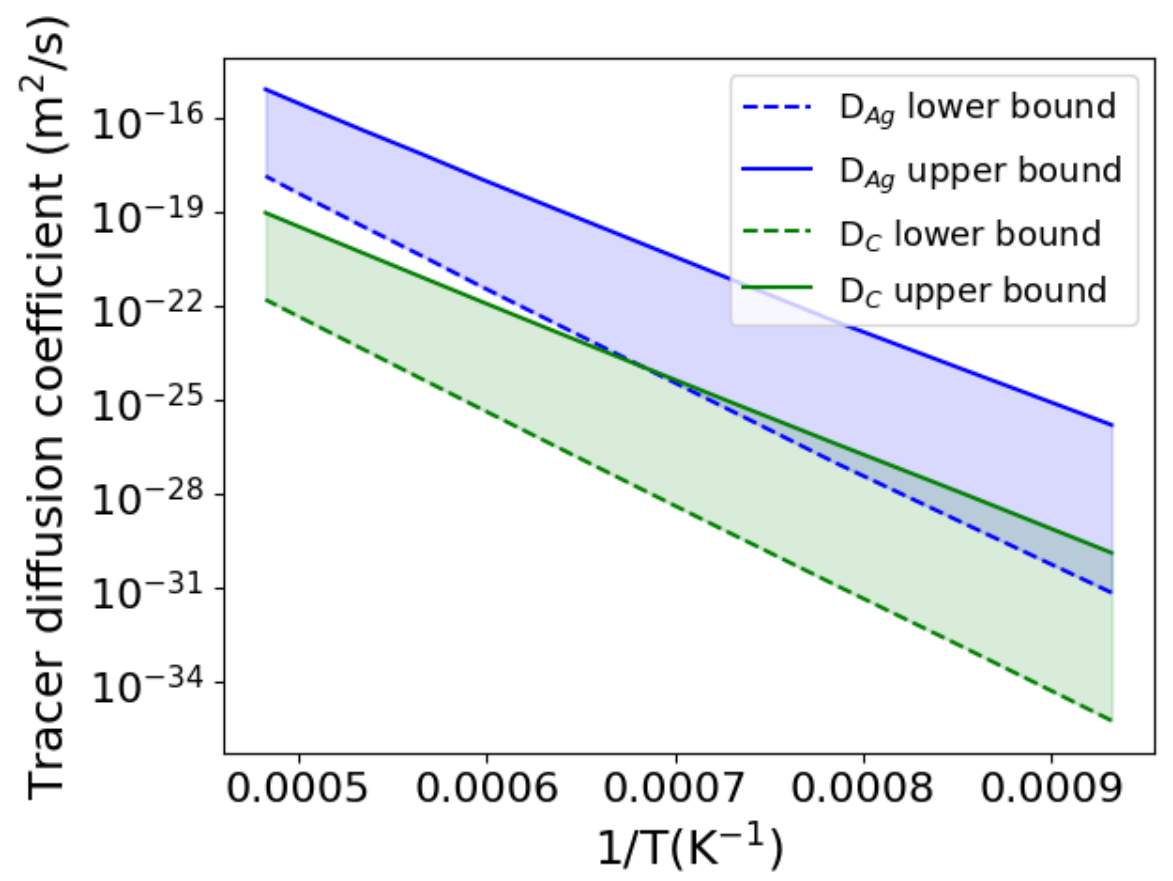

Figure 5: Plots showing the upper and lower bounds of the tracer diffusion coefficients of Ag and $\mathrm{C}$ in $\mathrm{SiC}$ as a function of $1 / \mathrm{T}$.

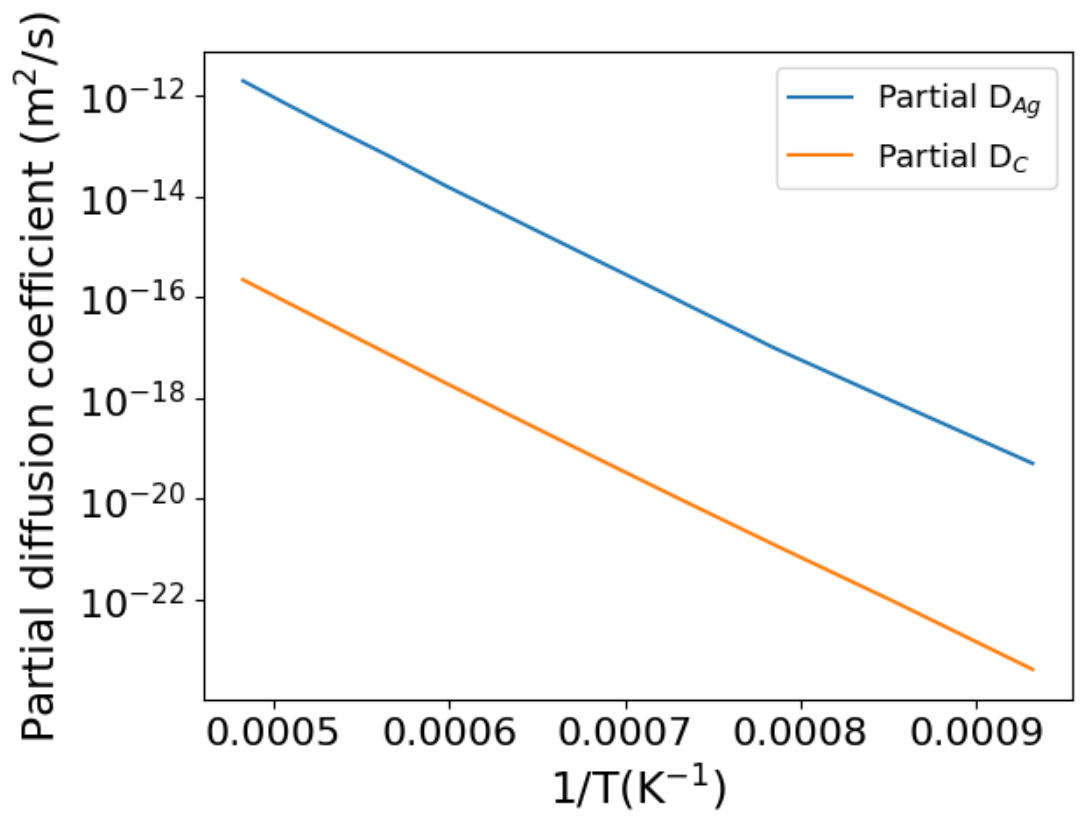

Figure 6: Plots showing the partial diffusion coefficients of $\mathrm{Ag}$ and $\mathrm{C}$ as a function of 1/T. 


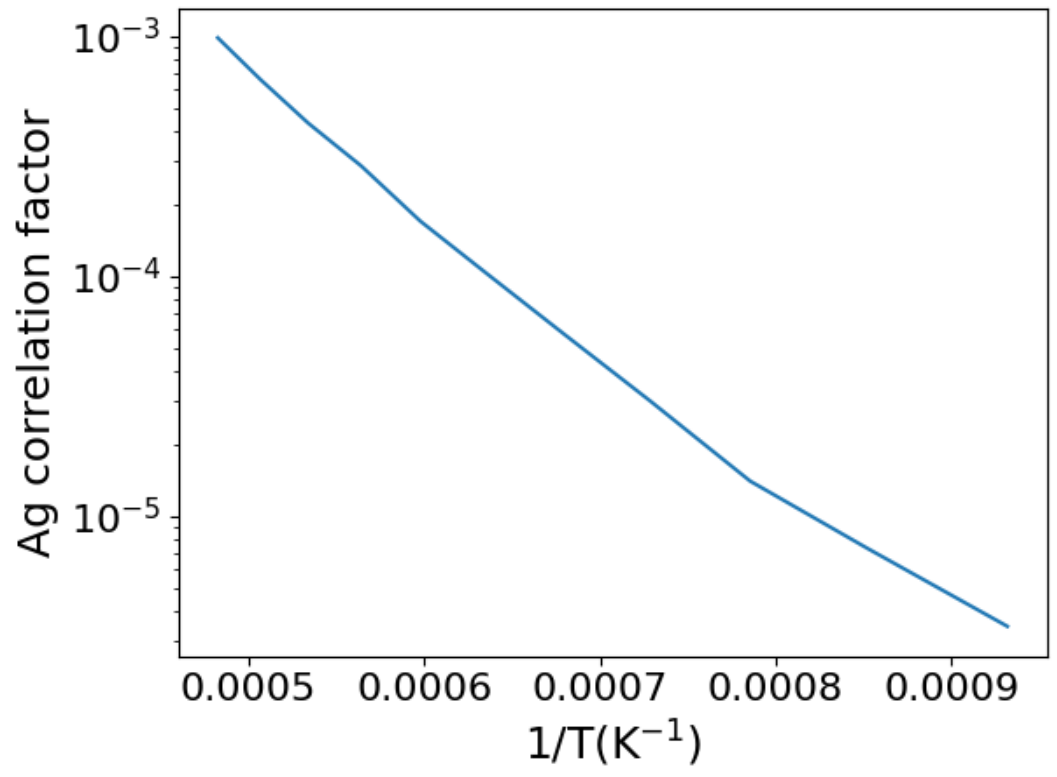

Figure 7: Plots showing the correlation factor of $\mathrm{Ag}$ diffusion in $\mathrm{SiC}$ as a function of 1/T. 


\section{MOLECULAR DYNAMICS SIMULATIONS OF Ag DIFFUSION IN SiC}

In this section, the tracer diffusivity of $\mathrm{Ag}$ along $\mathrm{SiC}$ GBs is obtained as a function of temperature using molecular dynamics (MD) simulations. For comparison, MD results for the diffusion of $\mathrm{Ag}$ interstitials in bulk crystalline $\mathrm{SiC}$ are also shown. Finally, the advanced temperature accelerated dynamics method is attempted to predict $\mathrm{Ag}$ diffusion in bulk amorphous $\mathrm{SiC}$, which was proposed as a model for high-energy grain boundaries [4].

\subsection{Ag Diffusion Along Grain Boundaries}

We have performed classical MD simulations in the Large-scale Atomic/Molecular Massively Parallel Simulator (LAMMPS) [19] to predict the average diffusivity of Ag atoms along GBs in polycrystalline $\mathrm{SiC}$ over a wide temperature range from $2000 \mathrm{~K}$ to $3000 \mathrm{~K}$. The analytical bond-order potential (ABOP) developed by Chen et al. [20] was employed to describe the interactions between silver and silicon carbide. Using the Voronoi tessellation method, we have constructed two periodic simulation cells for two-dimensional (2-D) and three-dimensional (3-D) SiC polycrystals, respectively (see Fig. 8). The $2-\mathrm{D}$ cell has a dimension of $15 \mathrm{~nm} \times 15 \mathrm{~nm} \times 2.2 \mathrm{~nm}$, while the 3 -D cell has a dimension of $10 \mathrm{~nm} \times 10 \mathrm{~nm} \times 10 \mathrm{~nm}$. The 3 -D cell contains $47727 \mathrm{Si}$ atoms, $47686 \mathrm{C}$ atoms, and 8 grains with random positions and orientations. The 2-D cell contains $23485 \mathrm{Si}$ atoms and $23515 \mathrm{C}$ atoms. All 4 grains in the 2-D model have their [001] axes aligned with the $\mathrm{z}$ direction.

To simulate the $\mathrm{Ag}$ diffusion in polycrystalline $\mathrm{SiC}$, we have inserted 123 and $496 \mathrm{Ag}$ atoms in the GB regions of the 2-D and 3-D simulation cells, respectively. The local atomic concentrations of $\mathrm{Ag}$ at the GB regions in the 2-D and 3-D cells have been calculated to be $2.7 \%$ and $2.8 \%$, respectively. To obtain the equilibrium cell dimensions at a given temperature, MD simulations in the NPT (constant number of atoms, pressure and temperature) ensemble are first performed for 10 ps. Long-time MD trajectories (up to $100 \mathrm{~ns}$ ) in the NVT (constant number of atoms, volume and temperature) ensemble are then collected and used to extract the mean squared displacement (MSD) data for Ag atoms as follows:

$$
M S D(t)=\frac{1}{N_{A g}} \sum_{i=1}^{N_{A g}}\left|R_{i}(t)-R_{i}(0)\right|^{2}
$$

Finally, the tracer diffusivity of Ag atoms can be determined from the Einstein relation as:

$$
D_{A g}^{G B}=\lim _{t \rightarrow \infty} \frac{M S D(t)}{6 t}
$$

Our MD calculated MSD data for Ag atoms diffusing along GBs in 2-D and 3-D SiC polycrystals are shown in Fig. 9. From the slopes of MSD vs. time data, the Ag diffusivity can be calculated using Eq. 3. By fitting our MD results in the temperature range from $2000 \mathrm{~K}$ to $3000 \mathrm{~K}$ to Arrhenius equation, we have obtained the following equation to describe the temperature-dependent diffusivity of $\mathrm{Ag}$ along $\mathrm{SiC}$ GBs:

$$
D_{A g}^{G B}=1.813 \times 10^{-7} \exp \left(-\frac{2.178}{k_{B} T}\right)\left(\mathrm{m}^{2} / \mathrm{s}\right)
$$

As shown in Fig. 10, Eq. 4 can explain Ag diffusion in both 2-D and 3-D SiC polycrystals reasonably well.

It is worth noting that all $\mathrm{Ag}$ atoms remain in the grain boundary region of the 2-D and 3-D $\mathrm{SiC}$ polycrystals throughout the MD simulations. No diffusion of $\mathrm{Ag}$ atoms back into the bulk 
region has been observed. This is consistent with our molecular statics calculations showing that $\mathrm{Ag}$ interstitials have a very strong tendency to segregate to grain boundaries in $\mathrm{SiC}$ (see Fig. 11).

\subsection{Interstitial Ag Diffusion in Bulk Crystalline SiC}

The high formation energy calculated for interstitial Ag atoms in Section 2.2 suggests that in thermodynamic equilibrium conditions, interstitial Ag will not contribute significantly to overall diffusion of Ag. However, under irradiation, the concentration of Ag interstitials may be significantly higher than at equilibrium. Therefore, using the same method as described in Section 3.1, we have further performed MD simulations to study the diffusion of Ag interstitials in bulk crystalline SiC. For this purpose, a cubic simulation cell containing $16384 \mathrm{Si}$ atoms, $16384 \mathrm{C}$ atoms, and 82 $\mathrm{Ag}$ atoms have been generated, in which the Ag atoms randomly occupy the tetrahedral interstitial sites of the $\mathrm{SiC}$ lattice that are surrounded by four carbon atoms. The atomic concentrations of $\mathrm{Ag}$ in bulk $\mathrm{SiC}$ is thus $0.25 \%$. Our MD calculated MSD data are shown in Fig. 12a. Interestingly, while $\mathrm{Ag}$ interstitials can initially migrate very rapidly on the bulk SiC lattice via the tetrahedralto-tetrahedral path, their diffusion slows down significantly with increasing time, as shown by the plateaus in the MSD vs. time curves in Fig. 12a. Fig. 13 further shows four MD snapshots for Ag interstitial diffusion in bulk crystalline $\mathrm{SiC}$ at $\mathrm{t}=0 \mathrm{~ns}, 1 \mathrm{~ns}, 10 \mathrm{~ns}$, and $60 \mathrm{~ns}$. It can be observed that single $\mathrm{Ag}$ interstitials in bulk $\mathrm{SiC}$ have a strong tendency to cluster with each other to form dimers, trimers, and larger Ag aggregates. Our MD simulations further show that Ag interstitial clusters larger than dimers are immobile within MD time scale (see Fig. 12b).

The MD predicted agglomeration of $\mathrm{Ag}$ interstitials in $\mathrm{SiC}$ is not only fully consistent with synchrotron X-ray absorption fine-structure spectroscopy (XAFS) experiments [21] suggesting that $\mathrm{Ag}$ within $\mathrm{SiC}$ layer is of metallic character, but is also the origin of the significant slowdown of $\mathrm{Ag}$ bulk diffusion in $\mathrm{SiC}$. From thermodynamic point of view, since $\mathrm{Ag}$ has essentially zero solubility in $\mathrm{SiC}$, the small $\mathrm{Ag}$ interstitial clusters can act as precursors for the formation of large Ag precipitates, which have been observed during Ag implantation experiments [8].

\subsection{Modeling Ag Diffusion in Bulk SiC Using Temperature-accelerated Dynamics}

To study Ag diffusion mechanisms in crystalline and amorphous $\mathrm{SiC}$, temperature accelerated dynamics (TAD) implemented in LAMMPS was used. TAD is able to identify the migration path and barrier of a diffusion event. The simulation systems contain $1000 \mathrm{SiC}$ and $1 \mathrm{Ag}$ atoms. Fig. 14 shows TAD simulation of $\mathrm{Ag}$ interstitial diffusion in a crystalline $\mathrm{SiC}$ at $1500 \mathrm{~K}$. Ag interstitial mainly hops between different tetrahedral sites. The primary migration barriers for the hops are $1.023 \mathrm{eV}$ and $1.196 \mathrm{eV}$. Sometimes smaller barriers $(0.497 \mathrm{eV}$ and $0.526 \mathrm{eV})$ are also found, but they are likely due to some artificially metastable states predicted by the potential.

TAD was also used to study Ag diffusion in an amorphous SiC at $2000 \mathrm{~K}$ (see Fig. 15). Although $\mathrm{Ag}$ did diffuse some distance, no single barrier can be identified for the Ag diffusion, because there are many different barriers due to the structural relaxation of the amorphous solid. Therefore, TAD may not be useful for studying the Ag diffusion in the amorphous solids. 


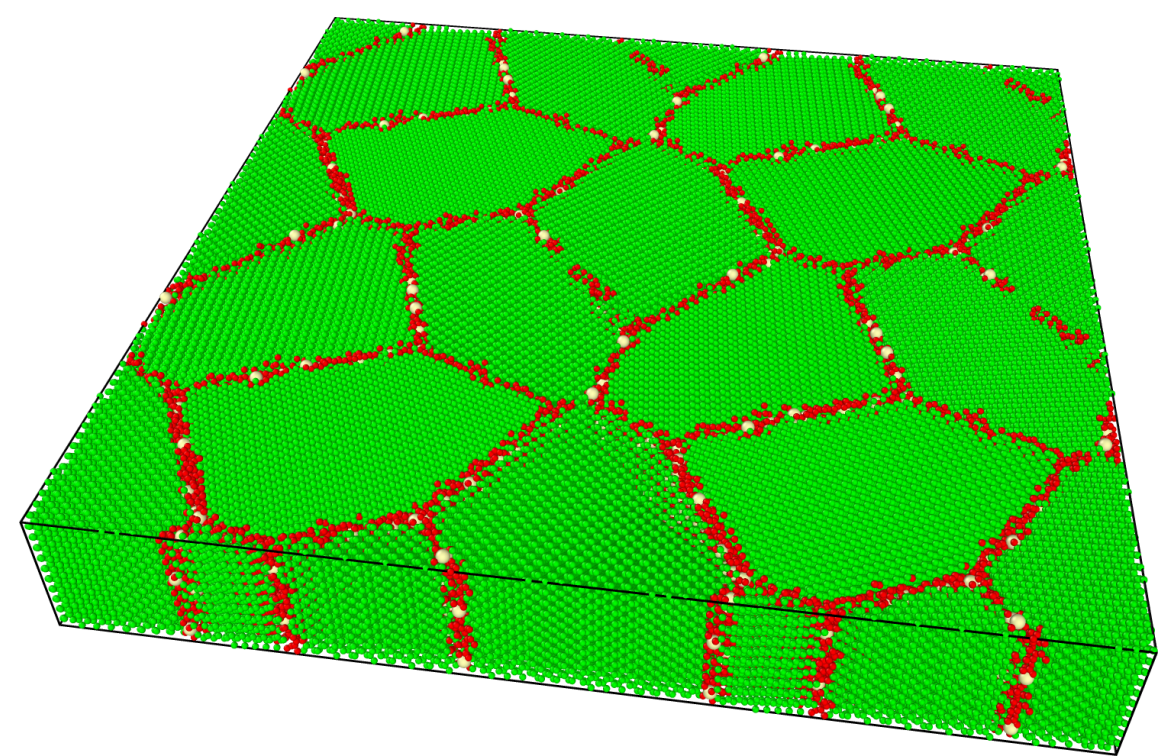

(a) $2-\mathrm{D}$

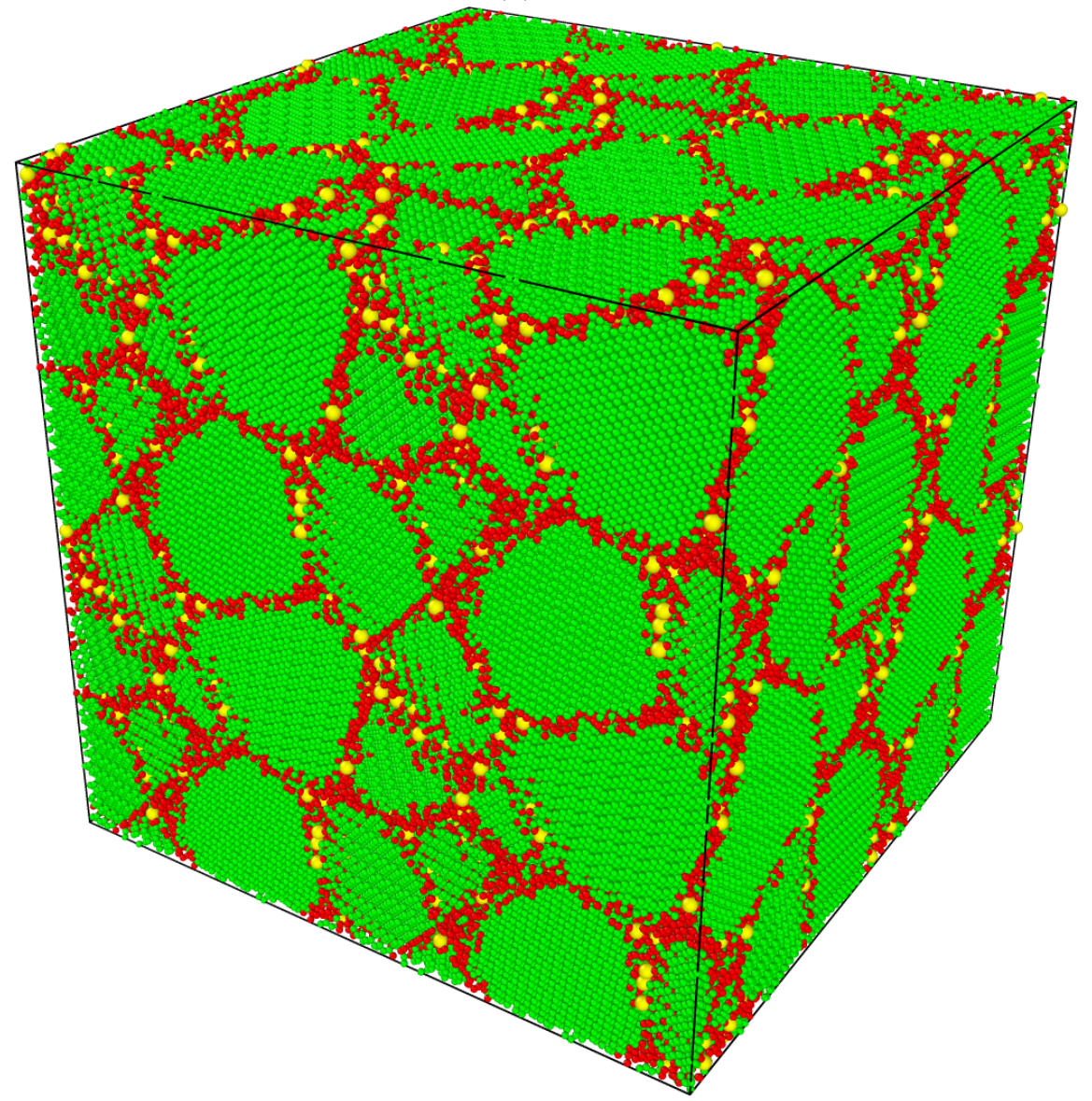

(b) 3-D

Figure 8: 2-D and 3-D polycrystalline $\mathrm{SiC}$ microstructures considered in this study. The green, red, and yellow spheres represent lattice $\mathrm{Si} / \mathrm{C}$ atoms, GB Si/C atoms, and Ag atoms, respectively. 


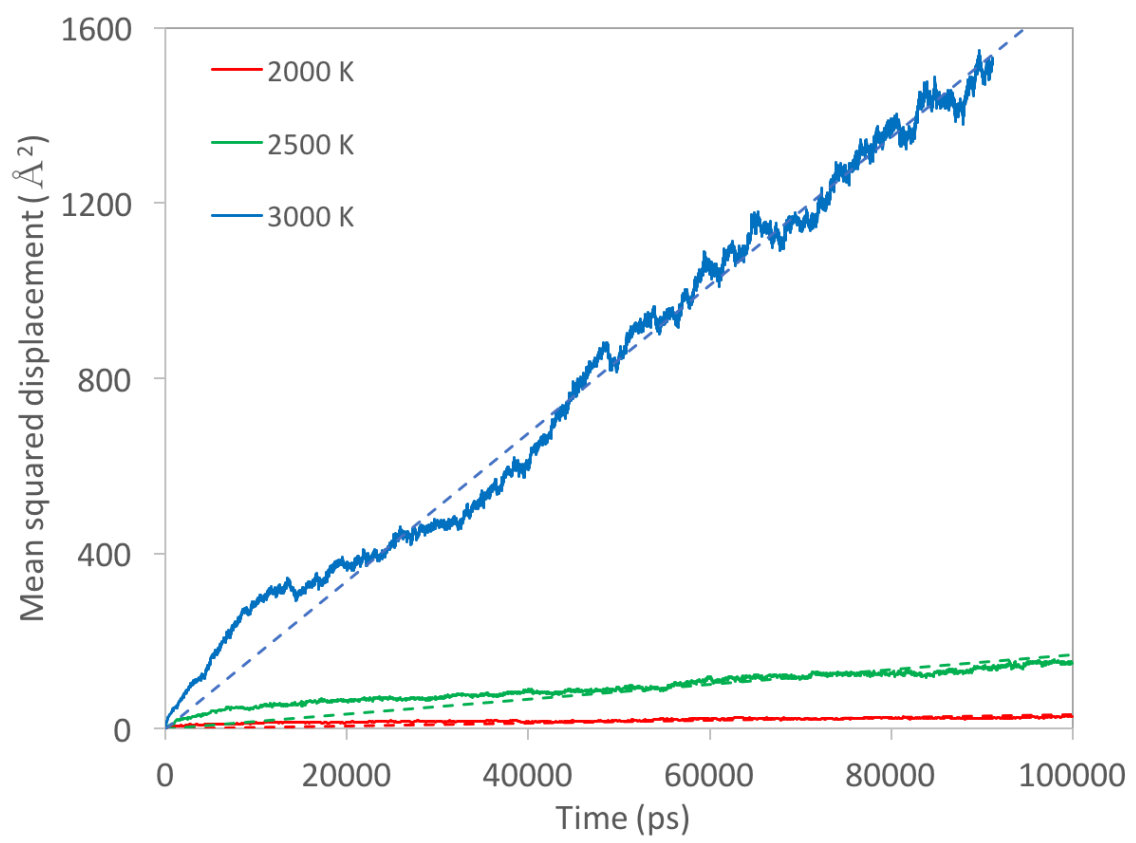

(a) $2-\mathrm{D}$

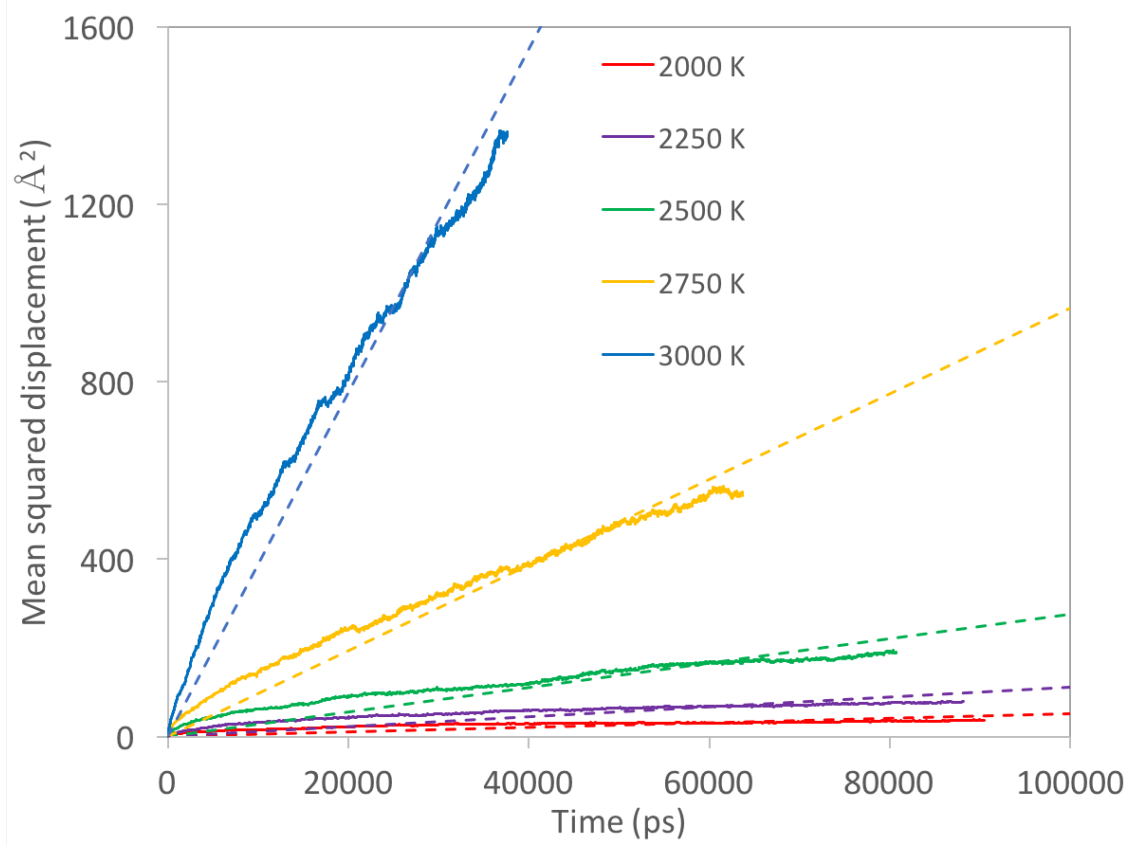

(b) 3 -D

Figure 9: Time-dependent mean squared displacements (MSD) of Ag atoms diffusing along GBs in 2-D and 3-D SiC polycrystals at various temperatures. The dashed lines represent linear fitting of MSD vs. time data. 


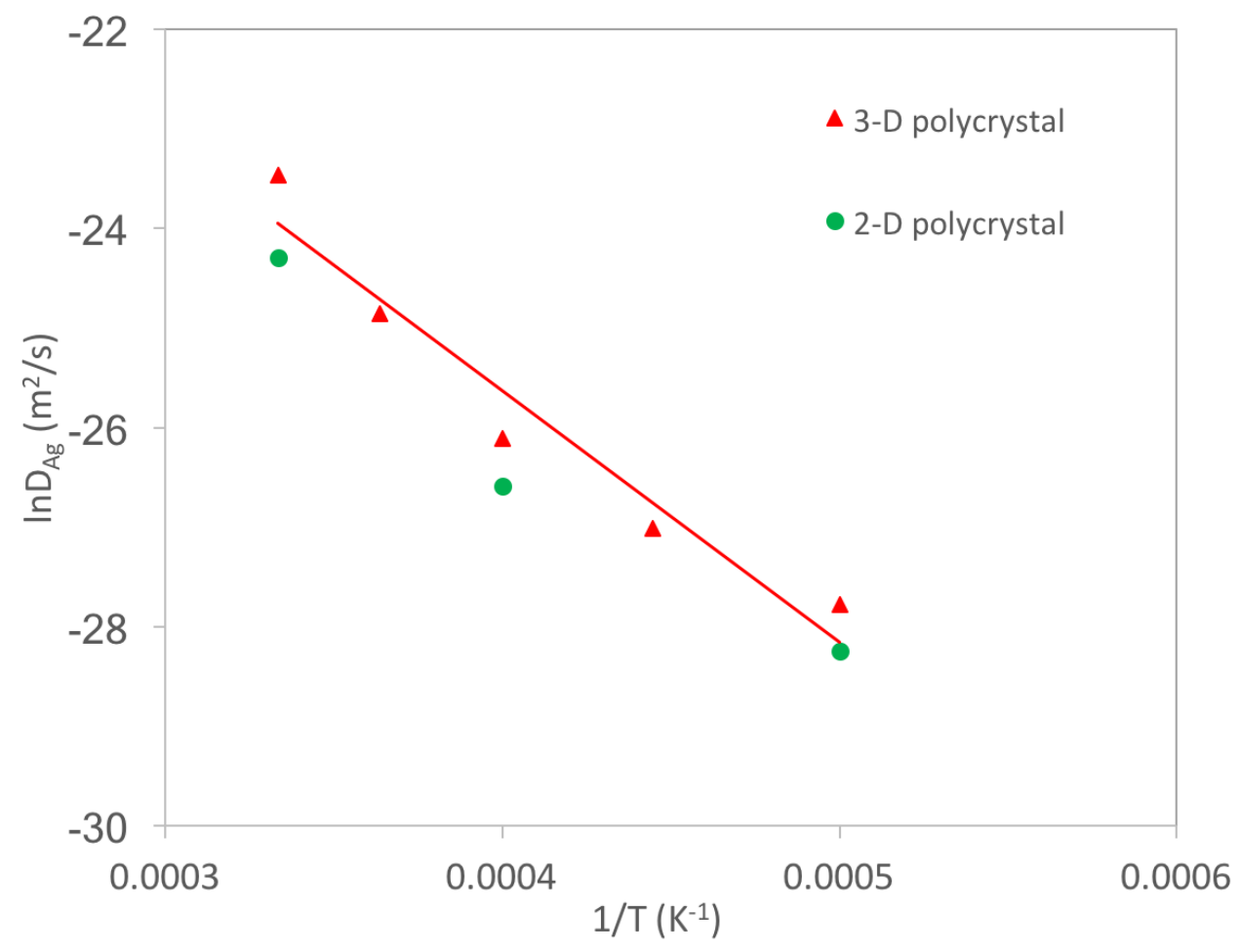

Figure 10: Arrhenius plot for GB diffusivity of $\mathrm{Ag}$ in SiC. The solid line represents the best fitting of MD calculated data using the Arrhenius equation.

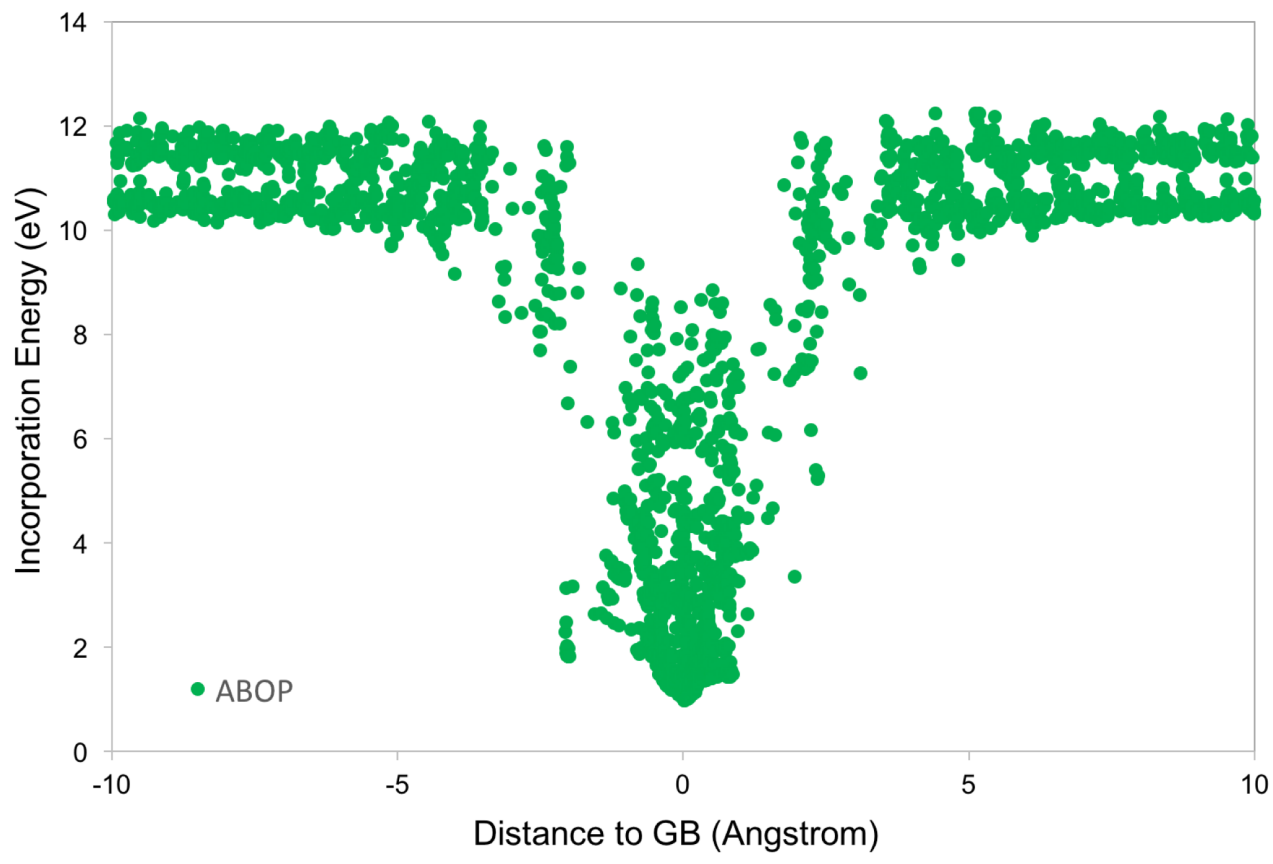

Figure 11: Formation energy of a Ag interstitial at various locations close to a GB in SiC. 


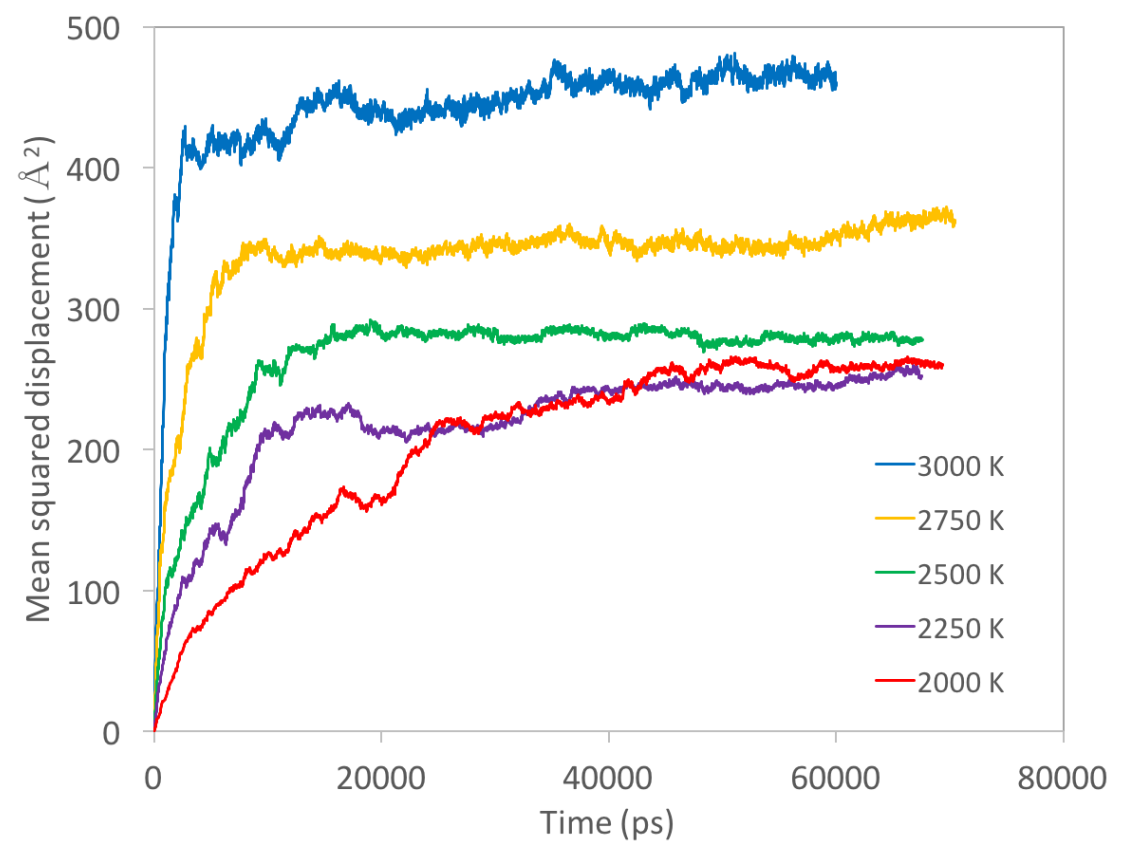

(a) Average MSD for all Ag atoms

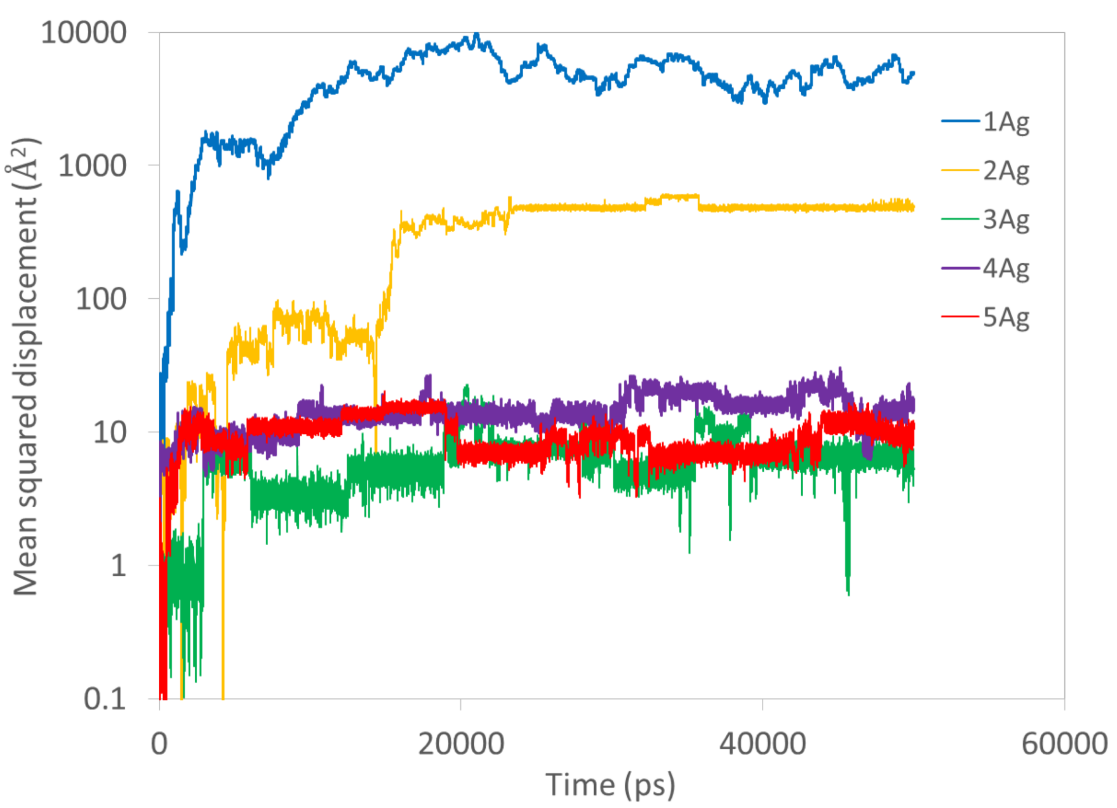

(b) MSD of individual Ag clusters at $2500 \mathrm{~K}$

Figure 12: Time-dependent MSD of Ag atoms diffusing in bulk crystalline $\mathrm{SiC}$ at various temperatures. 


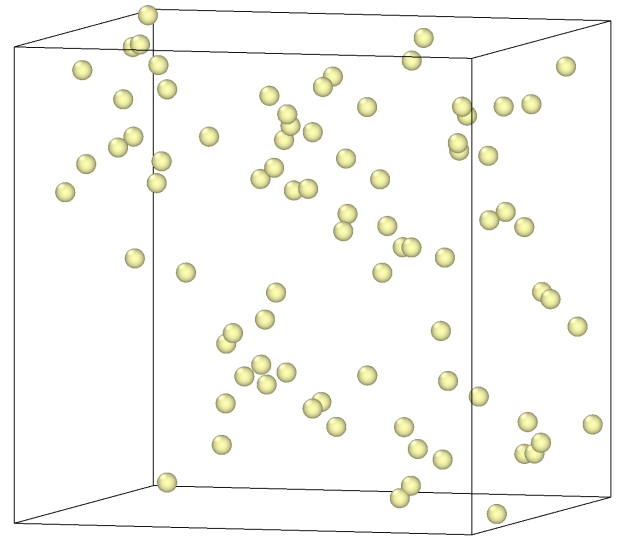

(a) 0 ns

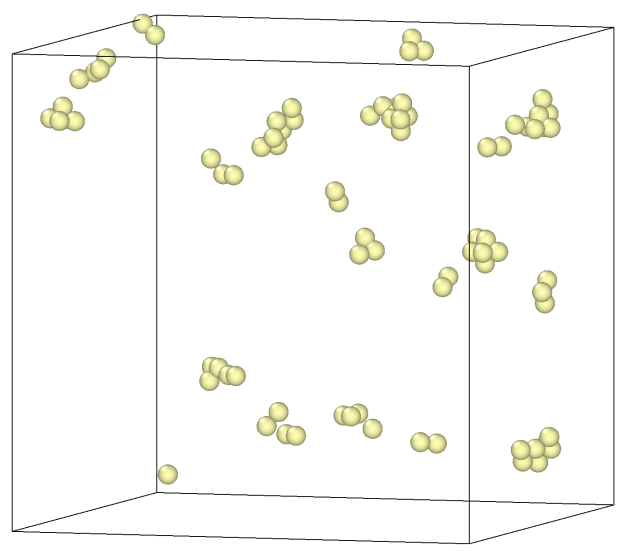

(c) $10 \mathrm{~ns}$

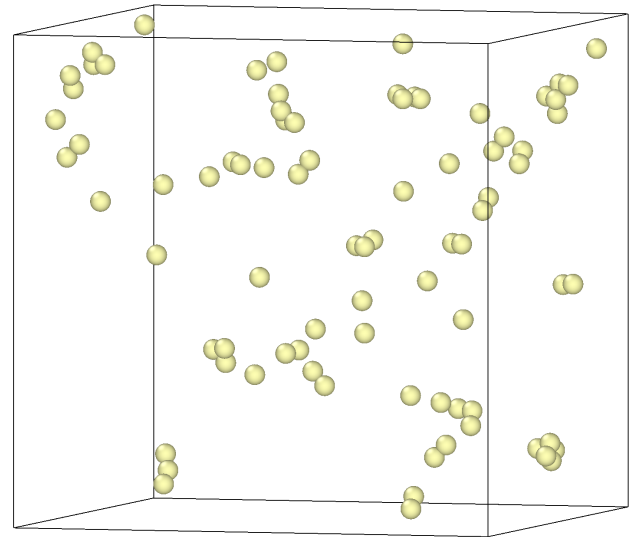

(b) $1 \mathrm{~ns}$

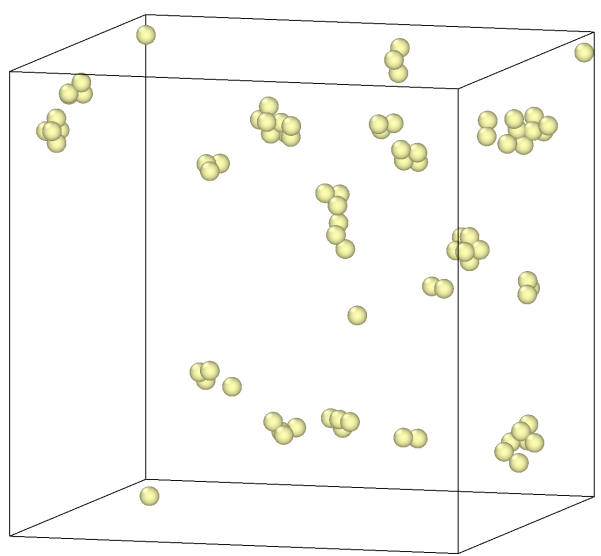

(d) $60 \mathrm{~ns}$

Figure 13: Snapshots of MD simulations of Ag interstitial diffusion in bulk crystalline SiC at 2500 K. For easy visualization, only the silver atoms are shown as yellow spheres.

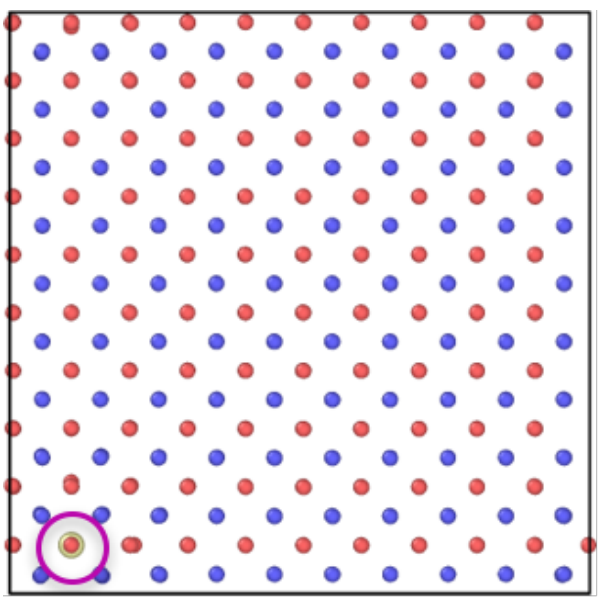

Figure 14: TAD simulation of Ag diffusion in a crystalline SiC. 

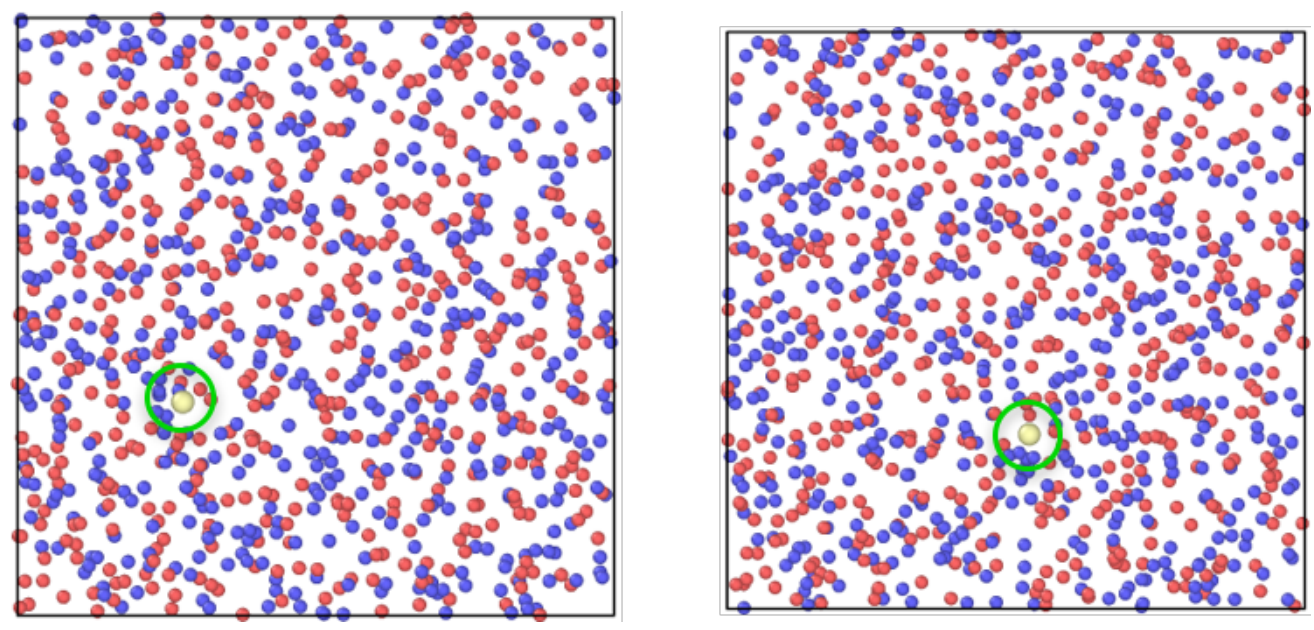

Figure 15: TAD simulation of Ag diffusion in amorphous SiC. 


\section{EFFECTIVE DIFFUSIVITY OF Ag VIA POLYCRYSTALLINE SiC}

It is hypothesized that the $\mathrm{SiC}$ microstructure influences the release of silver, as GBs can serve as pathways for $\mathrm{Ag}$ diffusion across the $\mathrm{SiC}$ layer [22, 23, 24]. As shown in Sections 2 and 3, diffusion of $\mathrm{Ag}$ along $\mathrm{SiC} \mathrm{GBs}$ is significantly quicker than in bulk SiC. Microstructure variables such as grain size, grain aspect ratio, grain shape, and GB types are therefore expected to affect Ag release out of the $\mathrm{SiC}$ layer. In this section, we use the diffusivity values in the $\mathrm{SiC}$ bulk and GBs provided by atomistic calculations in Sections 2 and 3 to investigate the effect of grain size and aspect ratio on effective $\mathrm{Ag}$ diffusion in the $\mathrm{SiC}$ layer.

One of the main challenges of leveraging atomistic calculations to provide an effective diffusivity at the mesoscale is the need to scale up the GB size. To simulate a larger microstructure that encompasses several grains, we need to increase the size of the GBs to reduce computational costs of the mesoscale model. In the case of $\mathrm{Ag}$ diffusion in $\mathrm{SiC}$, a simulation cannot resolve the 1-nmthick GBs in a domain that is several hundreds of $\mu \mathrm{m}$ large. Unfortunately, simply scaling up the GB size could artificially increase the importance of GB diffusion and lead to an overestimation of the effective Ag diffusivity. In this section, we derive different approaches to modify the bulk and GB diffusion coefficients of a system to predict an accurate, effective diffusivity when increasing GB size to perform mesoscale calculations.

The scale-up approaches are described and verified in Section 4.1. They are then used in Section 4.2 where the mesoscale effective Ag diffusivity is derived in polycrystalline SiC. To investigate the effect of grain size and grain aspect ratio, the effective $\mathrm{Ag}$ diffusivity is derived in several $\mathrm{SiC}$ polycrystals with varying microstructure properties corresponding to the $\mathrm{SiC}$ layer microstructure in Advanced Gas-cooled Reactor (AGR-1) TRISO fuel particles. Section 4.2 therefore provides the mesoscale effective $\mathrm{Ag}$ diffusivity in $\mathrm{SiC}$ as a function of temperature, grain size, and grain aspect ratio. This new insight on the effect of the $\mathrm{SiC}$ microstructure on $\mathrm{Ag}$ diffusion is then implemented in Bison in Section 4.3 to predict Ag release. For validation, Bison's predictions are compared against experimental measurements on AGR-1 TRISO fuel particles.

\subsection{Scaling Up of the Microstructure for Effective Diffusivity Calculations}

\subsubsection{Analytical Expression of the Effective Diffusion Coefficient for Simple Microstructures}

For simple microstructures, such as the ones shown in Fig. 16, it is possible to derive an analytical solution for the effective diffusion coefficient in both directions by using the analogy with a resistivity model. Along the $x$-axis of Fig. 16a, the diffusivity of the grains and grain boundaries act in parallel. The effective diffusivity is thus equal to

$$
D_{1, x}^{e f f}=\frac{L_{x}}{A_{x}} \frac{1}{R_{x}}=\frac{L_{x}}{A_{x}}\left(\frac{A_{b, x} D_{b}}{l_{b, x}}+\frac{A_{G B, x} D_{G B}}{l_{G B, x}}\right),
$$

with $D_{1, x}^{\text {eff }}$ the effective diffusion coefficient along the $x$-axis, $L_{x}$ the length of the domain along the $x$-axis, $A_{x}$ is the relevant area for mass transport along the $x$-axis (equal to $L_{y} \times L_{z}$ with $L_{y}$ the length of the domain along the $y$-axis, and $L_{z}$ the length of the domain in the $z$-axis), $R_{x}$ the resistance along the $x$-axis, and $A_{i, x}$ the relevant area for mass transport along the $x$-axis for phase $i, l_{i, x}$ the length of phase $i$ along the $x$-axis, and $D_{i}$ the diffusion coefficient in phase $i$, with $i=b$ for the bulk (inside of the grains), and $i=G B$ in the GB. A GB width of $w_{G B}$ leads to 
$A_{b, x}=\left(L_{y}-2 w_{G B}\right) L_{z}, A_{G B, x}=2 w_{G B} L_{z}$, and $l_{b, x}=l_{G B, x}=L_{x}$. Assuming a square domain with $L_{x}=L_{y}=L$, Eq. (5) becomes

$$
D_{1, x}^{e f f}=D_{b}+\frac{2 w_{G B}}{L}\left(D_{G B}-D_{b}\right)
$$

Similarly, the effective diffusion coefficient along the $y$-axis of Fig. 16 a $D_{1, y}^{e f f}$ can be derived by noting that the grains and grain boundaries acts in series. $D_{1, y}^{e f f}$ is thus equal to

$$
D_{1, y}^{e f f}=\frac{L_{y}}{A_{y}} \frac{1}{R_{y}}=\frac{L_{y}}{A_{y}}\left(\frac{l_{b, y}}{A_{b, y} D_{b}}+\frac{l_{G B, y}}{A_{G B, y} D_{G B}}\right)^{-1},
$$

where $L_{y}, A_{y}=L_{x} \times L_{z}, R_{y}, l_{b, y}, A_{b, y}, l_{G B, y}$, and $A_{G B, y}$ defined similarly than above, but for the $y$-axis. A GB width of $w_{G B}$ leads to $l_{b, y}=L_{y}-2 w_{G B}, l_{G B, y}=2 w_{G B}$, and $A_{b, y}=A_{G B, y}=A_{x}$. Assuming a square domain with $L_{x}=L_{y}=L$, Eq. (7) becomes

$$
D_{1, y}^{e f f}=\frac{D_{b} D_{G B}}{\left(1-\frac{2 w_{G B}}{L}\right) D_{G B}+\frac{2 w_{G B}}{L} D_{b}} .
$$

For the microstructure shown in Fig. 16b, there are two slightly different approaches to derive the effective diffusion coefficient. The first approach sees the microstructure as the horizontal GB in parallel with the perpendicular GB and the grain, which are in series. The other approach considers the microstructure as the horizontal GBs in series with the parallel GB and the grain, which are in parallel. The first approach provides, in the case of a square domain,

$$
D_{2 a}^{e f f}=\frac{L}{A} \frac{1}{R}=\frac{L}{A}\left(\frac{A_{2 a^{\prime}} D_{2 a^{\prime}}}{l_{2 a^{\prime}}}+\frac{A_{G B} D_{G B}}{l_{G B}}\right),
$$

with $A_{2 a^{\prime}}=L_{z} *\left(L-w_{G} B\right), A_{G B}=L_{z} * w_{G} B, l_{2 a^{\prime}}=l_{G B}=L$, and

$$
D_{2 a^{\prime}}=\frac{D_{b} D_{G B}}{\left(1-\frac{w_{G B}}{L}\right) D_{G B}+\frac{w_{G B}}{L} D_{b}} .
$$

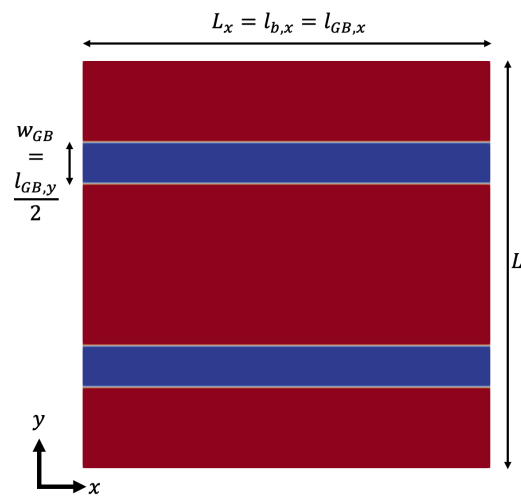

(a)

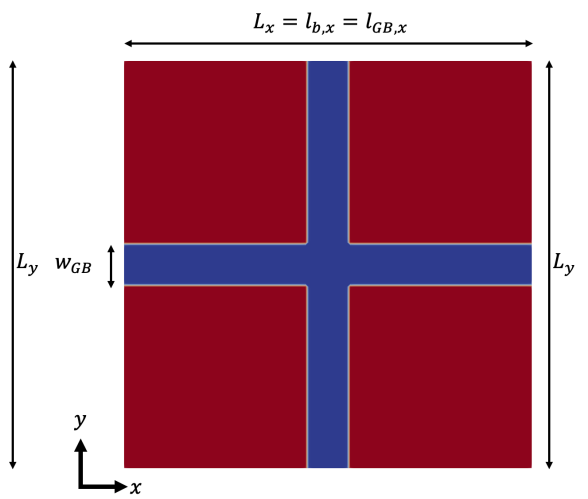

(b)

Figure 16: Simple periodic microstructures with two grain boundaries (blue) (a) along the $x$-axis, and (b) intersecting. $L_{x}$ and $L_{y}$ are the domain lengths along the $x$ and $y$-axis, respectively. $w_{G B}$ is the GB width. $l_{G B, x}$ and $l_{G B, y}$ are the length of he grain boundaries along the $x$ and $y$-axis, respectively, and $l_{b, x}$ is the length of the grain along the $x$-axis. 
Using the first approach, the effective diffusion coefficient for the microstructure shown in Fig. 16b is thus equal to

$$
D_{2 a}^{e f f}=\left(1-\frac{w_{G B}}{L}\right) \frac{D_{b} D_{G B}}{\left(1-\frac{w_{G B}}{L}\right) D_{G B}+\frac{w_{G B}}{L} D_{b}}+\frac{w_{G B}}{L} D_{G B}
$$

Similarly, the second approach leads to

$$
D_{2 b}^{e f f}=D_{G B} L \frac{\left(D_{b} L-D_{b} w_{G B}+D_{G B} w_{G B}\right)}{D_{b} L w_{G B}-D_{b} w_{G B}^{2}+D_{G B} L^{2}-D_{G B} L w_{G B}+D_{G B} w_{G B}^{2}}
$$

\subsubsection{Change in the Effective Diffusion Coefficient when the GB Width is Increased}

Eqs. (6), (8), (11), and (12) provide the effective diffusion coefficients for the microstructures shown in Fig. 16 as a function of the grain width $w_{G B}$ and the bulk and GB diffusion coefficients $D_{b}$ and $D_{G B}$. Because the effective diffusion coefficient depends on the material microstructure, it is important for the mesoscale model to simulate a representative section of the microstructure. This constraint often requires the use of an arbitrarily large $w_{G B}$, which can be larger than the physical value. In the following sections, we investigate several approaches that can be used to scale up the simulations.

The 'no change' approach: The 'no change' approach consists of increasing $w_{G B}$ without changing the other material parameters. With this approach, $D_{1, x}^{e f f, n c}\left(w_{G B}\right), D_{1, y}^{e f f, n c}\left(w_{G B}\right)$, and $D_{2}^{e f f, n c}\left(w_{G B}\right)$ are defined as in Eqs. (6), (8), (11), and (12) with $D_{b}$ and $D_{G B}$ defined constant. Unfortunately, as shown in Eq. (6), (8), (11), and (12), increasing $w_{G B}$ while keeping $D_{b}$ and $D_{G B}$ constant provides more importance to GB diffusion and is thus expected to influence the effective diffusion coefficients.

The 'constant product' approach The 'constant product' approach consists of keeping the $D_{G B} w_{G B}$ product a constant as $w_{G B}$ increases. As $w_{G B}$ increases, $D_{G B}$ decreases to compensate for the increases importance otherwise attributed to GB diffusion. The modified GB coefficient $D_{G B}^{c p}$ is thus defined as

$$
D_{G B}^{c p}=\frac{w_{G B}^{r e f}}{w_{G B}} D_{G B}
$$

with $w_{G B}^{r e f}$ the physical width of the GB, $D_{G B}$ the physical GB diffusion, and $w_{G B}$ the GB width used in the scaled up simulation. Injecting Eq. (13) into Eqs. (6), (8), (11), and (12) shows that, using this approach, $D_{1, x}^{e f f, c p}\left(w_{G B}\right), D_{1, y}^{e f f, c p}\left(w_{G B}\right), D_{2 a}^{e f f, c p}\left(w_{G B}\right)$, and $D_{2 b}^{e f f, c p}\left(w_{G B}\right)$ are still dependent of $w_{G B}$.

The 'analytical' approaches It is possible, however, to modify the bulk and GB diffusion coefficients $D_{b}$ and $D_{G B}$ as $w_{G B}$ increases to predict the same effective diffusion coefficients for specific configurations. For example, if $D_{b}^{m}, D_{G B}^{m}$, and $w_{G B}^{m}$ describe the modified parameters, then, based on Eqs. (6) and (8), the effective diffusion coefficients are accurately predicted for both directions of the microstructure shown in Fig. 16a during scale-up if and only if

$$
D_{1, x}^{e f f}=D_{b}+\frac{2 w_{G B}}{L}\left(D_{G B}-D_{b}\right)=D_{b}^{m}+\frac{2 w_{G B}^{m}}{L}\left(D_{G B}^{m}-D_{b}^{m}\right)
$$


and

$$
D_{1, y}^{e f f}=\frac{D_{b} D_{G B}}{D_{G B}-\frac{2 w_{G B}}{L}\left(D_{G B}-D_{b}\right)}=\frac{D_{b}^{m} D_{G B}^{m}}{D_{G B}^{m}-\frac{2 w_{G B}^{m}}{L}\left(D_{G B}^{m}-D_{b}^{m}\right)}
$$

are respected. For a given value of $w_{G B}^{m}$, this system can be solved for $D_{b}^{m}$ and $D_{G B}^{m}$.

Table. 2 shows different variants of this approach using different combinations of Eqs. (6), (8), (11), and (12). Five different approaches were developed, namely 'an 1x1y,' 'an 1x2a,' 'an 1y2a,' 'an 1x2b,' and 'an 1y2b.' These approaches are compared against each other and against the 'no change' and 'constant product' approaches in the next sections to determine the more accurate one.

Table 2: Different analytical approaches for scale up.

\begin{tabular}{ccc} 
Name & Eq. 1 & Eq. 2 \\
\hline 'an 1x1y' & $D_{1, x}^{\text {eff }}=D_{1, x}\left(w_{G B}^{m}\right)$ & $D_{1, y}^{\text {eff }}=D_{1, y}\left(w_{G B}^{m}\right)$ \\
'an 1x2a' & $D_{1, x}^{\text {eff }}=D_{1, x}\left(w_{G B}^{m}\right)$ & $D_{2 a}^{\text {eff }}=D_{2 a}\left(w_{G B}^{m}\right)$ \\
'an 1y2a' & $D_{1, y}^{\text {eff }}=D_{1, y}\left(w_{G B}^{m}\right)$ & $D_{2 a}^{\text {eff }}=D_{2 a}\left(w_{G B}^{m}\right)$ \\
'an 1x2b' & $D_{1, x}^{\text {eff }}=D_{1, x}\left(w_{G B}^{m}\right)$ & $D_{2 b}^{\text {eff }}=D_{2 b}\left(w_{G B}^{m}\right)$ \\
'an 1y2b' & $D_{1, y}^{\text {eff }}=D_{1, y}\left(w_{G B}^{m}\right)$ & $D_{2 b}^{\text {eff }}=D_{2 b}\left(w_{G B}^{m}\right)$
\end{tabular}

\subsubsection{Verification on Simple Bicrystals with Sharp Interfaces}

The performance of the approaches described above can be compared by deriving their error in the effective diffusion coefficients calculations for different values of $w_{G B}$ in the microstructure shown in Fig. 16. The percentage error can be determined as $D_{e r}^{e f f}=100 *\left(D^{e f f}\left(w_{G B}\right)-D^{e f f}\left(w_{G B}^{r e f}\right)\right) / D^{e f f}\left(w_{G B}^{r e f}\right)$ with $D^{e f f}\left(w_{G B}\right)$ being provided by the 'no change,' the 'constant product,' or the 'analytical' approaches. With the material parameters fixed as defined in Tab. 3, $D_{e r}^{e f f}$ was derived for each approach and is plotted in Fig. 17. Different ratios $D_{G B} / D_{b}$ were used to illustrate different heterogeneous cases.

Table 3: Parameters used to compare the errors in $D^{\text {eff }}$ from the different scaling approaches in Fig. 16.

\begin{tabular}{cccc}
$L$ & $w_{G B}^{r e f}$ & $D_{b}$ & $D_{G B} / D_{b}$ \\
\hline 1000 & 1 & $10^{-6}$ & $10 \rightarrow 10^{4}$
\end{tabular}

Results depicted in Fig. 17 show the 'analytical' approaches overall perform better than the other two approaches. The 'no change' approach drastically overpredicts $D^{e f f}$ for all four configurations, as expected. As discussed above, the 'constant product' approach predictions depend on $w_{G B}$, which is undesirable. It increasingly underestimates $D_{1, x}^{e f f}$ as $w_{G B}$ increases, and the scale of the error depends on the $D_{G B} / D_{b}$ ratio. The error in $D_{1, y}^{e f f}$ is more complex. For a low $D_{G B} / D_{b}$ ratio, $D_{1, x}^{e f f}$ is quickly underestimated. However, as $D_{G B} / D_{b}$ increases, $D_{1, x}^{e f f}$ can be significantly overestimated and predictions are as inaccurate as with the 'no change' approach. It follows that even if the 'constant product' can reasonably predict the contribution of parallel GBs for higher 


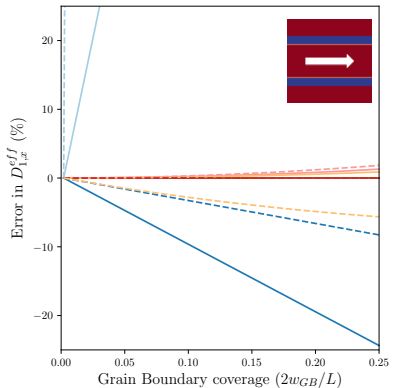

(a)

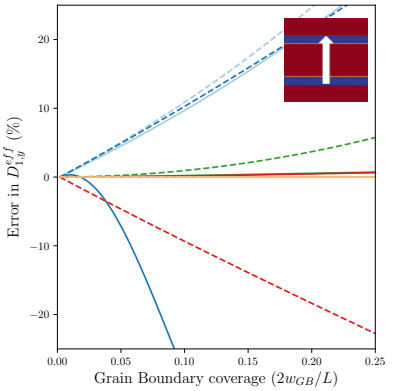

(b)

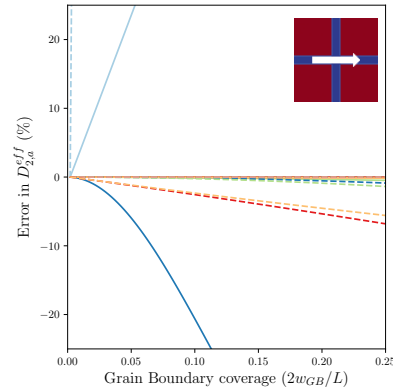

(c)

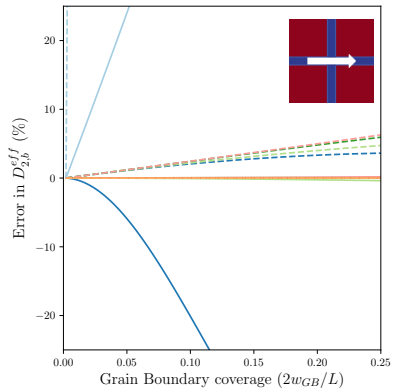

(d)

'no change' — 'constant product

'an $1 x 1 y$ '

'an $1 \times 2 a '$

'an $1 \mathrm{y} 2 \mathrm{a}$ '

'an $1 \mathrm{x} 2 \mathrm{~b}$

'an 1y2b'

$\longrightarrow D_{G B}=10 D_{b} \quad$---- $D_{G B}=10^{3} D_{b}$

Figure 17: Comparison of the percentage errors in effective diffusion coefficients derived by different scaling approaches applied to the microstructure shown in Fig. 16a along the (a) $x$-axis and the (b) $y$-axis, and in Fig. 16b using the approach (c) 2a and (d) 2b. The 'no change' approach significantly overestimates $D^{e f f}$ in all directions. The 'constant product' approach increasingly underestimates $D_{1, x}^{e f f}$ as $w_{G B}$ increases, and even more so for lower $D_{G B} / D_{b}$ values. Predictions for $D_{1, y}^{e f f}$ can also be as inaccurate as the 'no change' for large $D_{G B} / D_{b}$ values. The 'analytical' approaches, by definition, predict the same effective diffusion coefficient independently of $w_{G B}$ for the configurations it is based on. For the others, it performs better than the 'constant product' approach. The 'analytical 1x1y' approach consistently provides errors below $5 \%$ in the configurations studied.

$D_{G B} / D_{b}$ values, the contribution from GBs in series is expected to be significantly overestimated. Figures $17 \mathrm{c}$ and $17 \mathrm{~d}$ show that the 'constant product' approach tends to underpredict $D_{2 a}^{e f f}$ and $D_{2 b}^{e f f}$ for low $D_{G B} / D_{b}$ values as $w_{G B}$ increases. For high $D_{G B} / D_{b}$ values, $D_{2 a}^{e f f}$ is accurately predicted but $D_{2 b}^{e f f}$ is overpredicted.

The five 'analytical' approaches provide better results. By definition, the analytical approaches introduce no error for the configurations used to derive them. For example, the 'an 1x1y' approach introduces no error for $D_{1, x}^{e f f}$ and $D_{1, y}^{e f f}$. As shown in Fig. 17, when used to determine $D_{2 a}^{e f f}$ and $D_{2 b}^{e f f}$, the magnitude of the error is maintained below $2 \%$ and $5 \%$, respectively, for the cases tested. Similarly, 'an 1x2a,' 'an 1y2a,' 'an 1x2b,' and 'an 1y2b' provide better or comparable results compared to the 'constant product' approach. Their percentage error is, however, sometimes higher than for 'an 1x1y,' which seems to overall perform best on all the cases tested here.

\subsubsection{Verification on Polycrystals with Continuous Interfaces}

A method based on the approach described in Section 2.4.5.1 of Ref. [25] was used to generate polycrystals with desired grain sizes, grain aspect ratios, and GB widths. Some of these polycrystals are shown in Fig. 18, where the results of the verification are presented.

\subsubsection{Effective Diffusivity Calculations}

To determine the effective diffusivity coefficient, we use the Asymptotic Expansion Homogenization method described in Ref. [26]. This method is already implemented in MOOSE, making it possible to determine effective material properties in a domain with periodic boundary conditions. In the 


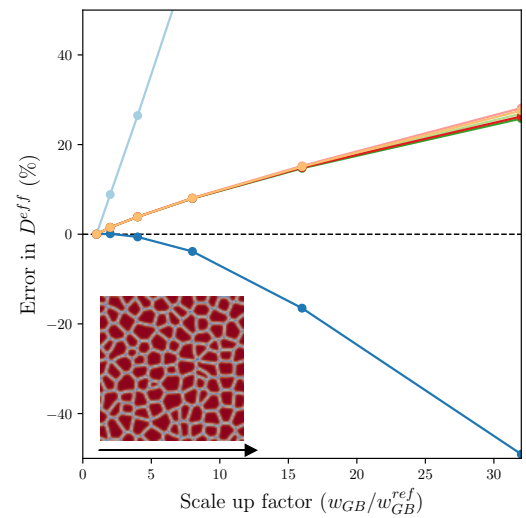

(a)

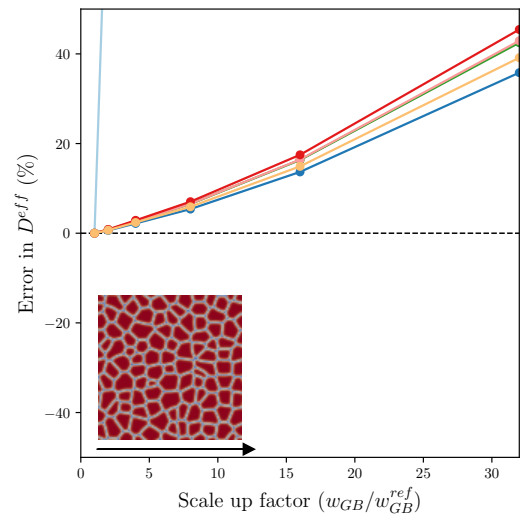

(d)

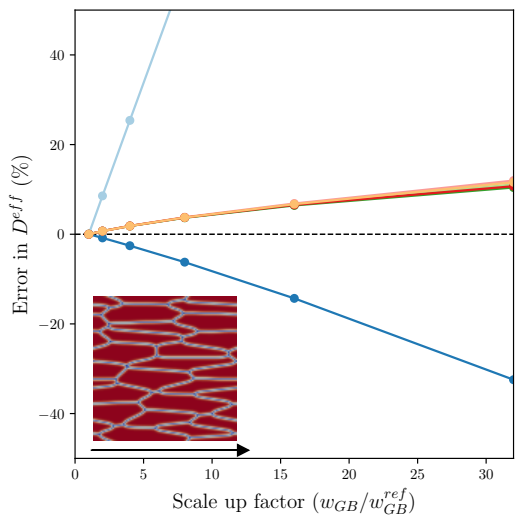

(b)

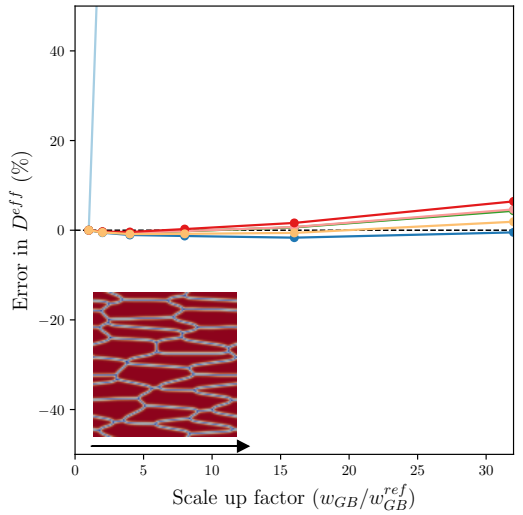

(e)

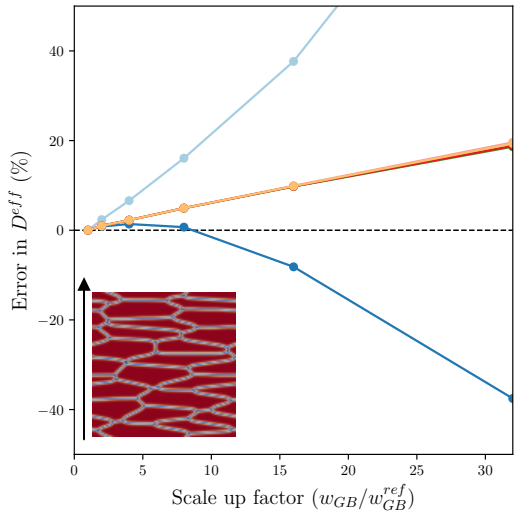

(c)

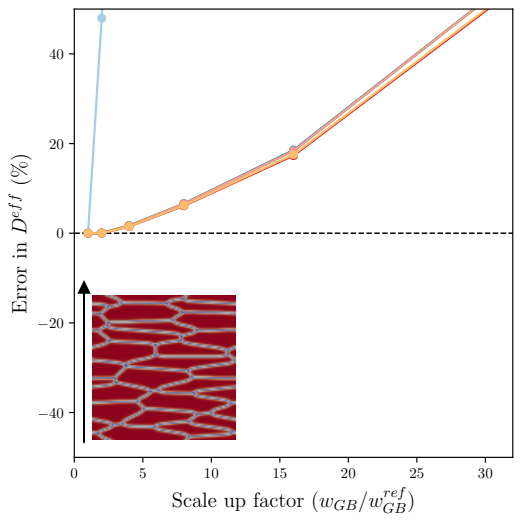

(f)

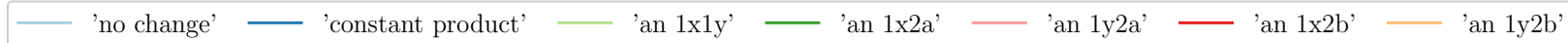

Figure 18: Verification of the scale-up approaches on polycrystalline microstructures with continuous interfaces. (a,b,c) show the error in the prediction of $D^{e f f}$ for two different microstructures with different grain elongations for $D_{G B} / D_{b}=10$. (c) differs from (b) in the direction for which $D^{e f f}$ was derived, as shown by the arrow. (d,e,f) show the results for the same study, but with $D_{G B} / D_{b}=10^{3}$. The introduction of the continuous interfaces and the more complex microstructures lead to greater errors than in Fig. 17. However, the error remains lower than $30 \%$ for the cases studied for low $D_{G B} / D_{b}$ ratio (a,b,c). For higher $D_{G B} / D_{b}$ ratio (d,e,f), $D^{e f f}$ can be overestimated by $40 \%$ for equiaxed grains. Remarkably, the predictions are very accurate along elongated grain (e) which is the case of interest for $\mathrm{Ag}$ diffusion in SiC. In all cases, each analytical approach provides similar predictions. Domain size is $1000 \times 1000$.

current work, the polycrystals were created periodic, and the temperature is considered constant across the entire domain. This method therefore applies.

\subsubsection{Results}

Figure 18 shows the results of the verification of the scale-up approaches on polycrystalline microstructures with continuous interfaces. The introduction of the continuous interfaces and the more complex microstructures lead to greater errors than in Fig. 17. However, the error remains lower than $30 \%$ for the cases studied for low $D_{G B} / D_{b}$ ratio. For higher $D_{G B} / D_{b}$ ratio, $D^{e f f}$ can 


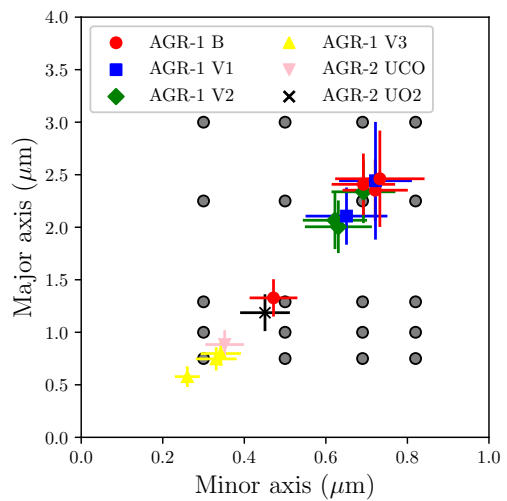

(a)

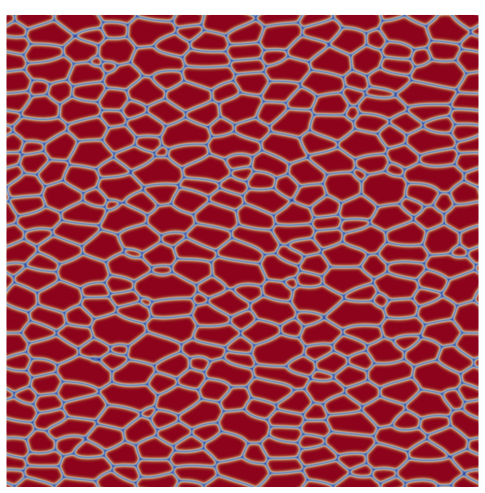

(b)

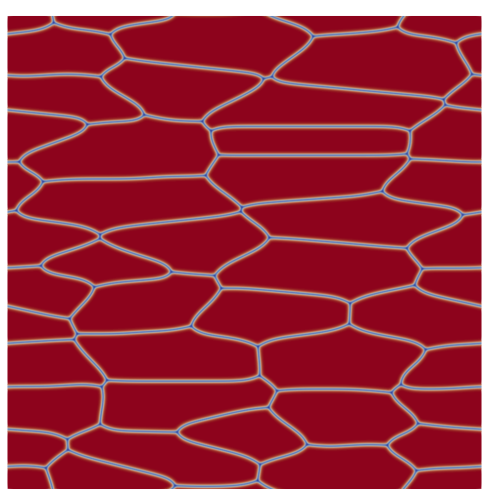

(c)

Figure 19: (a) Simulated grain dimensions (black dots) compared against the AGR-1 grain dimensions with twins included for all batches provided by Ref. [27]. The simulated microstructures cover the variations in grain dimensions found in the AGR-1 fuel batches. (b) and (c) show two examples of polycrystalline $\mathrm{SiC}$ microstructures created for this study, corresponding to the (b) bottom left and (c) top right points shown in (a). Domain size is $900 \mu \mathrm{m} \times 900 \mu \mathrm{m}$, and GBs are shown in blue and white.

be overestimated by $40 \%$ for equiaxed grains. Remarkably, the predictions are very accurate along elongated grain (e) which is the case of interest for $\mathrm{Ag}$ diffusion in $\mathrm{SiC}$. In all cases, all the analytical approaches provide similar predictions. Considering these results, the effective Ag diffusion coefficient in polycrystalline $\mathrm{SiC}$ will be derived by taking the average prediction of the analytical approaches for scale up.

\subsection{Mesoscale effective Ag diffusivity in polycrystalline SiC 4.2.1 Method: Generate Polycrystalline SiC}

As in Section 4.1.4, a method based on the approach described in Section 2.4.5.1 of Ref. [25] was used to generate polycrystalline $\mathrm{SiC}$ with desired grain sizes, grain aspect ratios, and GB widths. 2D domains of dimensions $9 \mu \mathrm{m} \times 9 \mu \mathrm{m}$ were created with grain major axis varying from $0.75 \mu \mathrm{m}$ to $3 \mu \mathrm{m}$, and grain minor axis varying from $0.3 \mu \mathrm{m}$ to $0.82 \mu \mathrm{m}$. Figure 19 shows the values of the microstructure variables from AGR-1 as experimentally measured by Gerczak et al. [27], as well as the simulated microstructures. The simulated microstructures cover the variations in grain dimensions found in the AGR-1 fuel batches. The GB size of the polycrystalline $\mathrm{SiC}$ is approximately equal to $1 \mathrm{~nm}$. However, as discussed above, the computational cost of the polycrystalline mesoscale simulations would be too great to resolve such a thin GB. The simulated polycrystalline $\mathrm{SiC}$ were thus defined with a $\mathrm{GB}$ width of $60 \mathrm{~nm}$.

\subsubsection{Method: Effective Diffusivity Calculations}

As described in Section 4.1.5, the Asymptotic Expansion Homogenization method detailed in Ref. [26] was used to derive the effective $\mathrm{Ag}$ diffusivity in polycrystalline $\mathrm{SiC}$ based on the bulk and GB diffusion coefficient provided by atomistic simulations. The analytical scale up approaches described in Section 4.1 are used to accurately predict the effective diffusivity while scaling up the 
GB width from $1 \mathrm{~nm}$ to $60 \mathrm{~nm}$. Calculations of the effective Ag diffusion coefficient are performed for different temperatures $(1450 \mathrm{~K}, 1505 \mathrm{~K}, 1800 \mathrm{~K}$, and $2073 \mathrm{~K})$ and for the microstructures with various grain sizes and aspect ratios shown in Fig. 19. This approach provides the effective Ag diffusivity as

$$
D^{e f f}\left(T, m_{i}, m_{a}\right)=D_{0}\left(m_{i}, m_{a}\right) \exp \left(-\frac{Q\left(m_{i}, m_{a}\right)}{R T}\right)
$$

with $T$ the temperature, $m_{i}$ the grain minor axis length, and $m_{a}$ the grain major axis length.

\subsubsection{Results}

The effective $\mathrm{Ag}$ diffusion coefficient $D^{\text {eff }}$ has to be fitted for three independent variables. First, the coefficients $D_{0}\left(m_{i}, m_{a}\right)$ and $Q\left(m_{i}, m_{a}\right)$ are derived for each microstructure by fitting $D^{e f f}$ as a function of temperature. This step provides a value for $D_{0}\left(m_{i}, m_{a}\right) Q\left(m_{i}, m_{a}\right)$ for each microstructure. The results are shown in Figs. 20(a-d). $D_{0}\left(m_{i}, m_{a}\right)$ appears independent of $m_{a}$, but seems to slightly depend on $m_{i}$, as shown in Figs. 20(a,b). The effect of $m_{i}$ is rather small and the quality of the linear fit is low, but $D_{0}\left(m_{i}, m_{a}\right)$ will still be fitted as

$$
D_{0}\left(m_{i}, m_{a}\right)=D_{0}\left(m_{i}\right)=D_{m, 0}+m_{i} D_{m, 1},
$$

with $D_{m, 0}=1.10^{9} \mathrm{~m}^{2} / \mathrm{s}, D_{m, 1}=-2.45^{-4} \mathrm{~m} / \mathrm{s}$, and $m_{i}$ in meters. $Q\left(m_{i}, m_{a}\right)$ is also independent of $m_{a}$ and increases linearly with increasing grain minor axis length, as shown in Figs. 20(c,d). It is therefore fitted as

$$
Q\left(m_{i}, m_{a}\right)=Q\left(m_{i}\right)=Q_{m, 0}+m_{i} Q_{m, 1},
$$

with $Q_{m, 0}=215455 \mathrm{~J} / \mathrm{mol}, Q_{m, 1}=1.74^{10} \mathrm{~J} / \mathrm{mol} / \mathrm{m}$, and $m_{i}$ in meters.

Based on the results shown in Fig. 20 and Eqs. (16) and (18), the effective Ag diffusion coefficient in $\mathrm{SiC}$ as a function of temperature and grain size is thus given as

$$
D^{e f f}\left(T, m_{i}\right)=\left(D_{m, 0}+m_{i} D_{m, 1}\right) \exp \left(-\frac{Q_{m, 0}+m_{i} Q_{m, 1}}{R T}\right)
$$

with $T$ in Kelvin, $m_{i}$ in meters, and $D_{m, 0}, D_{m, 1}, Q_{m, 0}$, and $Q_{m, 1}$ provided above. Remarkably, the effective diffusivity depends only on temperature and on the grain size in the direction perpendicular to the diffusion, and increases as $m_{i}$ decreases. This is because $m_{i}$ is inversely proportional to the number of GBs across the microstructure, which serve as preferential pathways in the case of $\mathrm{Ag}$ diffusion in polycrystalline SiC. Between the maximum and the minimum minor grain sizes used in this work, $D^{e f f}\left(T, m_{i}\right)$ increases by a factor up to 2.4 at these temperatures. The grain size in the direction parallel to the diffusion does not play a significant role, as it does not affect the number of pathways across the microstructure.

It is possible to compare the effective Ag diffusivity derived in this study with the current diffusivity value used in Bison. Figure 21 shows the bulk, GB, and the calculated effective diffusivities as functions of temperatures along with the value used in Bison. For the effective diffusivity, two values are provided for the extreme values of the minor axis length used in this study. The effective Ag diffusivity derived from the multiscale approach described in this study is slightly under the empirical Ag diffusivity currently in use in Bison. The activation energies of these two diffusivities, however, are very similar. It is possible to bring the effective Ag diffusivity at the level of the Bison diffusivity by adding a corrective factor $\delta_{\text {corr }}$ in $D_{0}\left(m_{i}\right)$. As shown in Fig. 21 we found that using

$$
D^{e f f, c o r r}\left(T, m_{i}\right)=\delta_{\text {corr }}\left(D_{m, 0}+m_{i} D_{m, 1}\right) \exp \left(-\frac{Q_{m, 0}+m_{i} Q_{m, 1}}{R T}\right)
$$




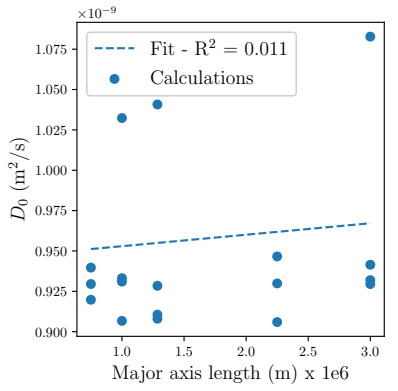

(a)

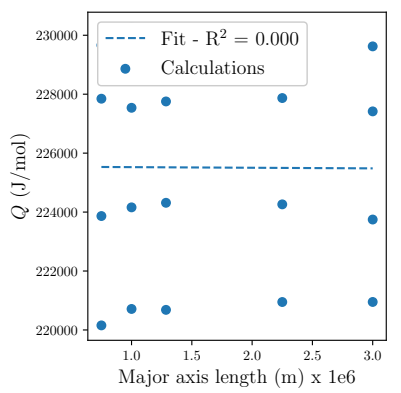

(c)

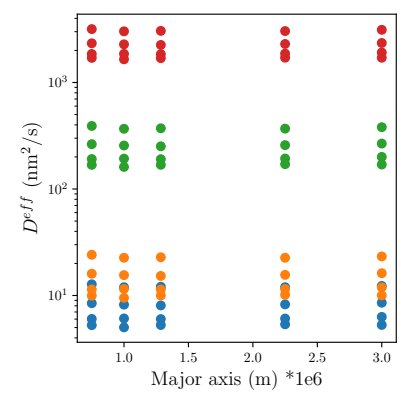

(e)

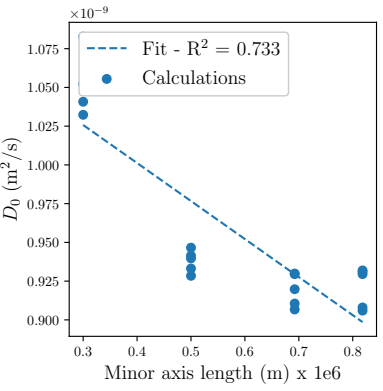

(b)

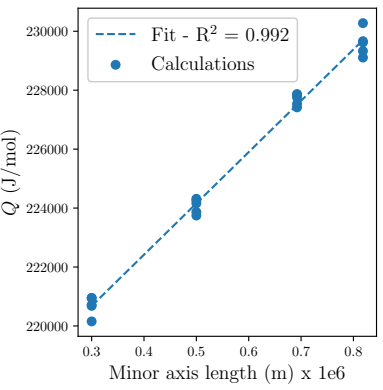

(d)

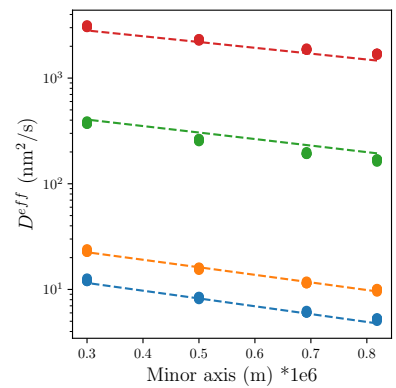

(f)

$-\mathrm{T}=1450 \mathrm{~K} \quad \mathrm{~T}=1505 \mathrm{~K} \quad-\mathrm{T}=1800 \mathrm{~K} \quad-\mathrm{T}=2073 \mathrm{~K}$

Figure 20: Derivation of the effective Ag diffusion coefficient as a function of the temperature $T$, the grain minor axis length $m_{i}$, and the grain major axis length $m_{a} .(\mathrm{a}, \mathrm{b})$ show how $D 0$ depends on (a) $m_{i}$ and (b) $m_{a}$. It seems to slightly decrease with increasing $m_{i}$. (c,d) show how $Q$ depends on (c) $m_{i}$ and (d) $m_{a}$. While $Q$ seems independent of $m_{a}$, it increases linearly with the $m_{i}$. (e,f) show how the effective diffusion coefficient $D^{e f f}$ depends on (e) $m_{i}$ and (f) $m_{a}$ for different temperatures. The best fit using the average value for $D_{0}$ and the linear fit for $Q\left(m_{i}\right)$ was also added on (e,f). The fit successfully captures the effects of temperature and microstructure on $D^{e f f}$.

with $\delta_{\text {corr }}=8.5$ as a substitute for the effective diffusion provided in Eq. (19) was enough to obtain a similar diffusivity to the empirical diffusivity while still accounting for the effect of the microstructure. 


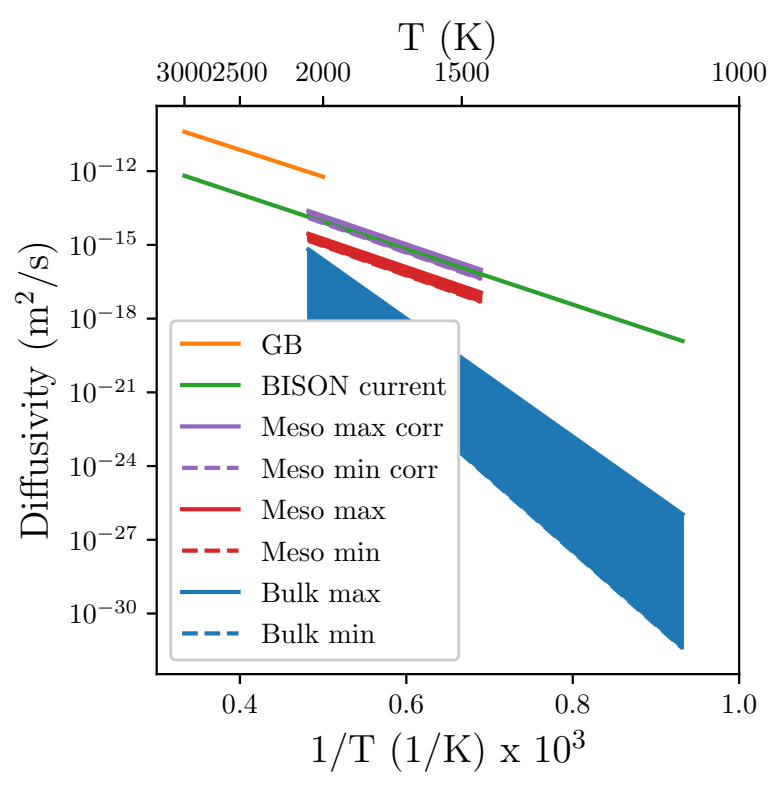

Figure 21: Comparison of the Ag diffusion coefficients provided by atomistic simulations for the bulk $D_{b u l k}$ and the GBs $D_{G B}$, the coefficient previously used in Bison $D_{B i s o n}$ provided in Ref. [1], and the effective diffusivity values provided by the current mesoscale study $D^{\text {eff }}$. The different values for $D^{\text {eff }}$ at each temperatures correspond to the extreme grain minor axis length values of the $\mathrm{SiC}$ microstructures described in Fig. 19. The effective Ag diffusion coefficient derived through atomistic and mesoscale calculations falls close to the empirical value currently used in Bison. Multiplying $D_{0}\left(m_{i}\right)$ by a corrective factor $\delta_{c o r r}=8.5$ is enough to increase the calculated effective $\mathrm{Ag}$ diffusivity to the same level than the empirical value used in Bison while still accounting for the effect of the microstructure, as shown by $D^{e f f, c o r r}$.

\subsection{Implementation of the Effective Ag Diffusivity in Bison 4.3.1 Methods}

The expressions of the effective Ag diffusivity in SiC provided in Eqs. (19) and (20) have been implemented in Bison in an attempt to predict the Ag release fraction measured in AGR-1 [28]. Different microstructure values were provided for different compacts, as they contain different fuel variants. Table 4 combines the information found in Table 6 and Fig. 2 of Ref. [29] and Fig. 3 of Ref. [27] to link microstructure properties to different fuel capsules. Note that although the capsules 3 and 6 all contain the baseline fuel, different values were used for the grain minor axis length to represent the two groups shown in Fig. 19a [27]. Except for the added dependence in microstructure properties, simulations were performed as in Ref. [1] and the results are provided in Fig. 22.

\subsubsection{Results}

Figure 22 shows the comparison between the measured and computed $\mathrm{Ag}$ release fractions for AGR-1 compacts under base irradiation conditions. The empirical Ag diffusivity currently used in Bison predicts, on average, a release fraction of around 0.3 and loosely corresponds to measured values. However, it misses some of the variations between capsules, sometimes underestimating, 
Table 4: Microstructure properties of AGR-1 fuel capsules.

\begin{tabular}{c|ccccccc}
\hline Compact & $1-3-1$ & $3-2-1$ & $3-3-1$ & $3-2-3$ & $4-4-1$ & $4-1-2$ & $4-3-2$ \\
Fuel Variant & V3 & B & B & B & V3 & V3 & V3 \\
Grain minor axis size $(\mu \mathrm{m})$ & 0.3 & 0.71 & 0.71 & 0.71 & 0.3 & 0.3 & 0.3 \\
\hline Compact & $4-3-3$ & $4-4-3$ & $5-3-1$ & $5-1-3$ & $5-2-3$ & $5-3-3$ & $6-1-1$ \\
Fuel Variant & V3 & V3 & V1 & V1 & V1 & V1 & B \\
Grain minor axis size $(\mu \mathrm{m})$ & 0.3 & 0.3 & 0.69 & 0.69 & 0.69 & 0.69 & 0.47 \\
\hline Compact & $6-2-1$ & $6-4-1$ & $6-4-3$ & $4-1-1$ & $4-4-2$ & $5-2-1$ & $6-3-2$ \\
Fuel Variant & $\mathrm{B}$ & $\mathrm{B}$ & $\mathrm{B}$ & $\mathrm{V} 3$ & $\mathrm{~V} 3$ & $\mathrm{~V} 1$ & $\mathrm{~B}$ \\
Grain minor axis size $(\mu \mathrm{m})$ & 0.47 & 0.47 & 0.47 & 0.3 & 0.3 & 0.69 & 0.47 \\
\hline
\end{tabular}

sometimes overestimating the release fraction. The Ag release fraction computed with the effective $\mathrm{Ag}$ diffusion coefficient derived from atomistic and mesoscale simulations provided by this study in Eq. (19) is consistently underestimated. The underestimation of the Ag diffusivity in SiC noted in Fig. 21 leads to an underestimation of the Ag release fraction. However, the corrected effective Ag diffusion coefficient provided in Eq. (20) provide predictions of Ag release that can be compared with the current empirical Bison predictions. Due to the effect of microstructure on the effective $\mathrm{Ag}$ diffusivity, the predicted $\mathrm{Ag}$ release fraction decreases for $\mathrm{SiC}$ layers with thicker grains, while it increases for $\mathrm{SiC}$ layers with thinner grains. An improvement is observed for compacts 1-3-1, $3-2-1,3-3-1,3-2-3,4-3-2,4-3-3,5-3-1,5-3-3,6-1-1,6-2-1,6-4-1$, and 6-4-3. However, the quality of the predictions decreases for compacts $4-4-1,4-1-2,4-4-3,5-1-3,5-2-3$. This study therefore shows that the predictions of $\mathrm{Ag}$ release from TRISO fuel through can be improved by accounting for the effect of $\mathrm{SiC}$ microstructure on the effective Ag diffusivity.

\subsection{Summary}

In this section, the $\mathrm{Ag}$ diffusivities of the $\mathrm{SiC}$ bulk and $\mathrm{GBs}$ are used to derive an mesoscale effective diffusivity that depends on temperature and microstructure properties such as grain size. The first part focuses on developing different approaches to appropriately scale up the GB size in mesoscale simulations to reduce computational costs without overestimating the role of GB diffusion. The second part shows the effect of microstructure properties on the effective Ag diffusivity. We show that a microstructure with grains that are smaller in the direction perpendicular to diffusion promote faster diffusion. This is attributed to the fact that more GBs are then available to serve as pathways for Ag. The resulting effective diffusion coefficient therefore depends on temperature and microstructure properties.

This effective diffusivity appears to be an order of magnitude lower than the empirical diffusion coefficient currently used in Bison. This underestimation could be due to an underestimation of Ag diffusion in $\mathrm{SiC}$ bulk and $\mathrm{GB}$, which currently neglect the effect of irradiation, palladium-assisted silver diffusion, and other physics. Moreover, the current mesoscale model does not yet account for the high diffusivity of high-angle GBs, which further promote Ag diffusion. The new effective Ag diffusivity was implemented in Bison to predict Ag release fractions in ARG-1 fuel compacts, but consistently underpredicts it. A corrective multiplying factor was therefore introduced to bring the new effective Ag diffusivity to the same level as the empirical value used in Bison, while still accounting for the effect of the microstructure. The Ag release predicted using this corrected version of the effective Ag diffusivity is, for most compacts, more accurate than the empirical 


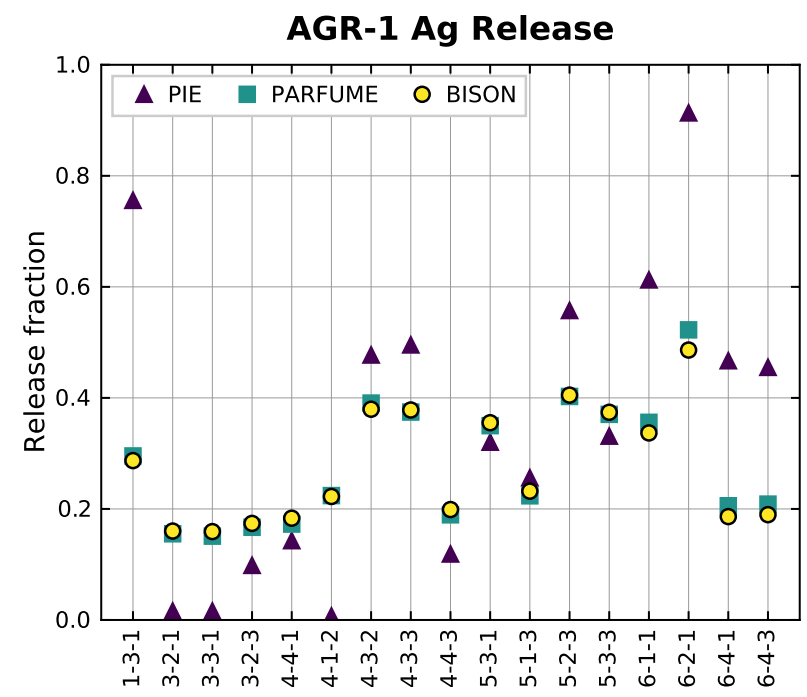

(a)

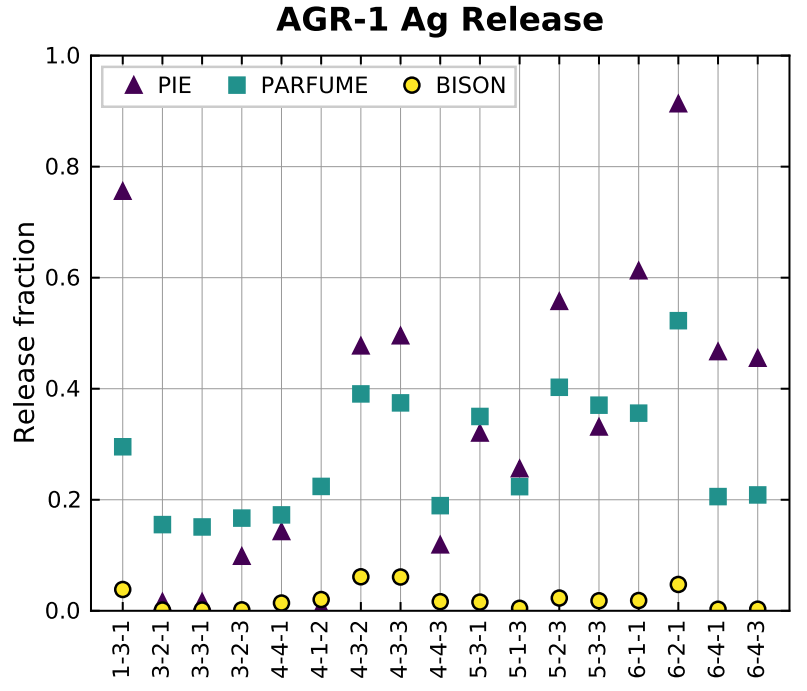

(b)
AGR-1 Ag Release

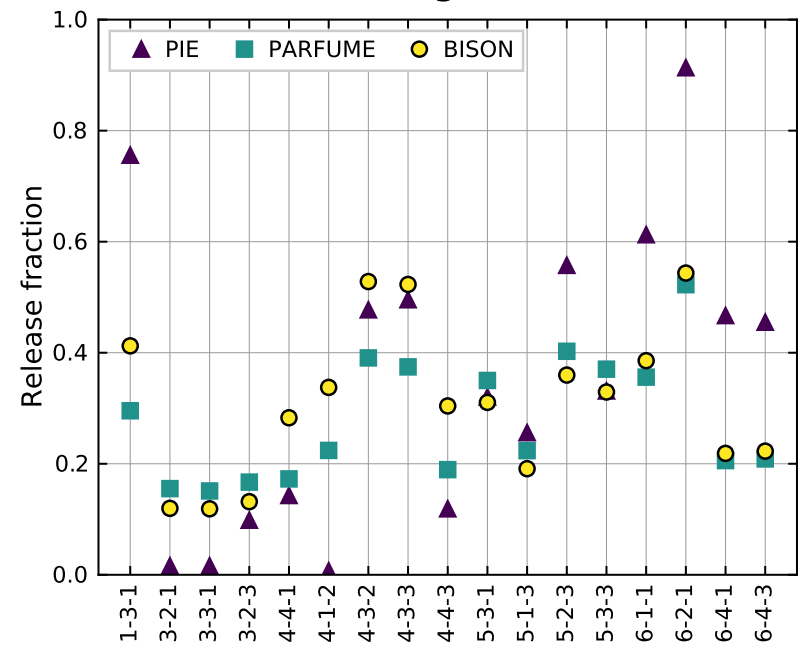

(c)

Figure 22: Comparison of measured (PIE) and computed (PARFUME, Bison) silver release fractions for AGR-1 compacts. (a) shows the results with the current empirical diffusivity currently in Bison, (b) with the effective Ag diffusion coefficient derived from multiscale approach derived in this study (Eq. (19)), (c) with the corrected effective Ag diffusion coefficient (Eq. (20)). The underestimation of the $\mathrm{Ag}$ diffusivity in $\mathrm{SiC}$ noted in Fig. 21 leads to an underestimation of the $\mathrm{Ag}$ release fraction. However, using the corrected effective Ag diffusion coefficient provides better predictions that can be compared with the current Bison model.

model currently used in Bison, thus showing the importance of accounting for the effect of SiC microstructure on Ag release. 


\section{Summary}

In FY-21, a multiscale approach combining ab initio calculations, molecular dynamics simulations, and phase-field simulations was employed to obtain the microstructure-dependent effective diffusivity of fission product $\mathrm{Ag}$ through the polycrystalline $\mathrm{SiC}$ layer in TRISO fuel particles. The temperature-dependent $\mathrm{Ag}$ diffusivities in bulk $\mathrm{SiC}$ and along $\mathrm{SiC}$ grain boundaries have been calculated from lower-length scale simulations, and the results are employed to parameterize a mesoscale model to predict the effective diffusion coefficients of $\mathrm{Ag}$ through $\mathrm{SiC}$ with a polycrystalline microstructure. Our major findings are summarized below:

(i) While an isolated $\mathrm{Ag}$ interstitial can migrate quickly in perfect $\mathrm{SiC}$ lattice, it can be deeply trapped by irradiation-induced $\mathrm{Si}$ and $\mathrm{C}$ vacancies to form substitutional $\mathrm{Ag}_{S i}$ and $\mathrm{Ag}_{C}$ defects. Among the four possible types of Ag-vacancy pairs $\left(\mathrm{Ag}_{S i}-\mathrm{Va}_{S i}, \mathrm{Ag}_{C}-\mathrm{Va}_{C}, \mathrm{Ag}_{S i}-\mathrm{Va}_{C}, \mathrm{Ag}_{C}-\mathrm{Va}_{S i}\right)$, the $\mathrm{Ag}_{C}-\mathrm{Va}_{C}$ complex is the most mobile and its diffusivity has been calculated using the kMC method. Both $\mathrm{Ag}_{S i}-\mathrm{Va}_{C}$ and $\mathrm{Ag}_{C}-\mathrm{Va}_{S i}$ defects relax to the same configuration, which is essentially immobile due to its high binding energy and rotation barriers.

(ii) Even without irradiation-induced vacancies, Ag interstitials have a strong tendency to cluster with each other to form dimers, trimers, and larger Ag interstitial clusters. Once self-trapping occurs, the mobilities of $\mathrm{Ag}$ interstitials in bulk $\mathrm{SiC}$ are significantly reduced. Ag interstitial clusters larger than dimers are found to be immobile within MD time scale.

(iii) The present molecular dynamics simulations suggest that $\mathrm{Ag}$ diffusion along $\mathrm{SiC}$ grain boundaries is orders-of-magnitude faster than bulk diffusion. Furthermore, Ag interstitials have a very strong tendency to segregate to grain boundaries in SiC. Grain boundary diffusion is thus the primary pathway for $\mathrm{Ag}$ penetration of the $\mathrm{SiC}$ layer.

(iv) The effective Ag diffusivity is significantly governed by the microstructure of SiC. A microstructure with grains that are smaller in the direction perpendicular to diffusion promotes faster diffusion. This is attributed to the fact that more grain boundaries are then available to serve as pathways for Ag. The calculated effective Ag diffusivity is within an order of magnitude lower than the empirical diffusion currently in use in Bison, and therefore underestimates the Ag release fraction from AGR-1 capsules. The discrepancy between the calculated and empirical diffusivity values may be caused by the fact that the bulk diffusivity calculations were perform using thermodynamic equilibrium defect concentrations, and irradiation effects on defect concentrations were not considered. Future work will address the effects of irradiation on diffusivity.

(v) A corrective multiplying factor was therefore introduced to bring the new effective $\mathrm{Ag}$ diffusivity to the same level compared to the empirical value used in Bison, while still accounting for the effect of the microstructure. For most capsules, the Ag release predicted using this corrected version of the effective Ag diffusivity is more accurate than the empirical model currently in use in Bison, thus showing the importance of accounting for the effect of $\mathrm{SiC}$ microstructure on $\mathrm{Ag}$ release. 


\section{References}

1. Jason D. Hales, Wen Jiang, Aysenur Toptan, and Kyle A. Gamble. Modeling fission product diffusion in triso fuel particles with bison. Journal of Nuclear Materials, 548:152840, 2021.

2. Johan B. Malherbe. Diffusion of fission products and radiation damage in sic. J. Phys. D: Appl. Phys., 46:473001, 2013.

3. T. M. Lillo and I. J. van Rooyen. Associations of pd, u and ag in the sic layer of neutronirradiated triso fuel. Journal of Nuclear Materials, 460:97-106, 2015.

4. Hyunseok Ko, J. Deng, I. Szlufarska, and D. Morgan. Ag diffusion in sic high-energy grain boundaries: Kinetic monte carlo study with first-principle calculations. Computational Materials Science, 121:248-257, 2016.

5. J. H. Neethling, J. H. O'Connell, and E. J. Olivier. Palladium assisted silver transport in polycrystalline sic. Nuclear Engineering and Design, 251:230-234, 2012.

6. E. J. Olivier and J. H. Neethling. The role of pd in the transport of ag in sic. Journal of Nuclear Materials, 432:252-260, 2013.

7. Robert A. Coward, Christopher R. Winkler, William A. Hanson, Michael L. Jablonski, and Mitra L. Taheri. Transmission electron microscopy investigation of ag diffusion mechanisms in $\beta$-sic. Journal of Nuclear Materials, 457:298-303, 2015.

8. H. J. MacLean, R. G. Ballinger, L. E. Kolaya, S. A. Simonson, N. Lewis, and M. E. Hanson. The effect of annealing at $1500{ }^{\circ} \mathrm{C}$ on migration and release of ion implanted silver in cvd silicon carbide. Journal of Nuclear Materials, 357:31-47, 2006.

9. H. Y. Xiao, Y. Zhang, L. L. Snead, V. Shutthanandan, H. Z. Xue, and W. J. Weber. Nearsurface and bulk behavior of ag in sic. Journal of Nuclear Materials, 420:123-130, 2012.

10. J. H. O'Connell and J. H. Neethling. Ag transport in high temperature neutron irradiated 3c-sic. Journal of Nuclear Materials, 445:20-25, 2014.

11. Izabela Szlufarska, Dane Morgan, and James Blanchard. Ag transport through non-irradiated and irradiated sic. 12016.

12. Sarah Khalil, N. Swaminathan, D. Shrader, A. Heim, D. Morgan, and I. Szlufarska. Diffusion of ag along $\sigma 3$ grain boundaries in 3c-sic. Physical Review B, 84, 2011.

13. D. Shrader, Sarah Khalil, T. Gerczak, T. Allen, A. Heim, I. Szlufarska, and D. Morgan. Ag diffusion in cubic silicon carbide. Journal of Nuclear Materials, 408:257-271, 2011.

14. M. Cooper, S. Kelly, and M. Bertolus. Atomic scale mobility of the volatile fission products xe, kr and i in cubic sic. Physical chemistry chemical physics : PCCP, 18 25:16902-11, 2016.

15. G. Henkelman, B. Uberuaga, and H. Jónsson. A climbing image nudged elastic band method for finding saddle points and minimum energy paths. Journal of Chemical Physics, 113:9901-9904, 2000 .

16. Fei Gao, W. J. Weber, M. Posselt, and V. Belko. Atomistic study of intrinsic defect migration in 3c-sic. Physical Review B, 69:245205, 2004. 
17. M. Bockstedte, Alexander Mattausch, and O. Pankratov. Ab initio study of the annealing of vacancies and interstitials in cubic sic: Vacancy-interstitial recombination and aggregation of carbon interstitials. Physical Review B, 69:235202, 2004.

18. G. Samolyuk, Y. Osetsky, and R. Stoller. Molecular dynamics modeling of atomic displacement cascades in 3c-sic: Comparison of interatomic potentials. Journal of Nuclear Materials, 465:8388, 2015.

19. S. Plimpton. Fast parallel algorithms for short-range molecular dynamics. J. Comput. Phys., 117:1-19, 1995.

20. Nanjun Chen, Qing Peng, Zhijie Jiao, Isabella van Rooyen, William F. Skerjanc, and Fei Gao. Analytical bond-order potential for silver, palladium, ruthenium and iodine bulk diffusion in silicon carbide. Journal of Physics: Condensed Matter, 32:085702, 2020.

21. Rachel L. Seibert, Kurt A. Terrani, Daniel Velazquez, John D. Hunn, Charles A. Baldwin, Fred C. Montgomery, and Jeff Terry. Local atomic structure of pd and ag in the sic containment layer of triso fuel particles fissioned to $20 \%$ burn-up. Journal of Nuclear Materials, 500:316-326, 2018.

22. D. A. Petti, J. Buongiorno, J. T. Maki, R. R. Hobbins, and G. K. Miller. Key differences in the fabrication, irradiation and high temperature accident testing of us and german triso-coated particle fuel, and their implications on fuel performance. Nuclear Engineering and Design, 222:281-297, 62003.

23. I J Van Rooyen, H Nabielek, J H Neethling, M J Kania, and D A Petti. Progress in solving the elusive ag transport mechanism in triso coated particles: "what is new?". Proceedings of the HTR 2014, Paper HTR2014-31261, 102014.

24. I. J. Van Rooyen, M. L. Dunzik-Gougar, and P. M. Van Rooyen. Silver (ag) transport mechanisms in triso coated particles: A critical review. Nuclear Engineering and Design, 271:180-188, 52014.

25. Pierre-Clément Simon. Phase field modeling and quantification of zirconium hydride morphology, Jan 2021.

26. J. D. Hales, M. R. Tonks, K. Chockalingam, D. M. Perez, S. R. Novascone, B. W. Spencer, and R. L. Williamson. Asymptotic expansion homogenization for multiscale nuclear fuel analysis. Computational Materials Science, 99:290-297, 32015.

27. Tyler J. Gerczak, John D. Hunn, Richard A. Lowden, and Todd R. Allen. Sic layer microstructure in agr-1 and agr-2 triso fuel particles and the influence of its variation on the effective diffusion of key fission products. Journal of Nuclear Materials, 480:257-270, 112016.

28. Blaise P. Collin, David A. Petti, Paul A. Demkowicz, and John T. Maki. Comparison of silver, cesium, and strontium release predictions using parfume with results from the agr-1 irradiation experiment. Journal of Nuclear Materials, 466:426-442, 112015.

29. J.D. Hunn, R.N. Morris, I. van Rooyen, T. Gerczak, J.M. Harp, S.A. Ploger, and P.A. Demkowicz. Agr-1 post irradiation examination final report, idaho national laboratory, 2015. 